\title{
Diversity and use of wild and non- cultivated edible plants in the Western Himalaya
}

Kamal Prasad Aryal ${ }^{1,3^{*}}$, Sushmita Poudel ${ }^{2}$, Ram Prasad Chaudhary ${ }^{1}$, Nakul Chettri $^{3}$, Pashupati Chaudhary ${ }^{4}$, Wu Ning ${ }^{3}$ and Rajan Kotru ${ }^{3}$

\begin{abstract}
Background: Local people in the Himalayan region use a wide range of wild and non-cultivated edible plants (WNEPs) for food, spice, medicinal, and cultural purposes. However, their availability, use, status and contribution to livelihood security are poorly documented, and they have been generally overlooked in recent agro-biodiversity conservation and management programmes. The study aimed to investigate WNEP diversity and current status in a part of the Kailash Sacred Landscape—a transboundary landscape shared by Nepal, India and PR China_in terms of collection, use, management and conservation initiatives.
\end{abstract}

Methods: Multiple methodologies and tools were used for data collection. A series of participatory tools (45 key informant interviews, 10 focus group discussions, a crop diversity fair, direct observation of species through a transect walk and rapid market assessments) was followed by a household survey (195 respondents) and complemented by a literature review.

Results: The study recorded 99 WNEPs belonging to 59 families of which 96 were angiosperms, one gymnosperm and two pteridophytes. Species were used for food, spice, medicine, rituals and income generation. Thirty-five species had multiple uses, including these: 40 species were used for fruit and 31 for vegetables. WNEPs contribute significantly to daily food requirements, especially the vegetables. The use value of Dryopteris cochleata was found highest (0.98) among frequently used vegetable species. The values of informant consensus factor were found maximum for worms in the stomach (0.99) and minimum for skin disease treatment (0.67). Nearly $85 \%$ of households depended exclusively on WNEPs for at least more than a month per year. Results on the importance and use of different species, gender roles in WNEP activities and conservation approaches are presented.

Conclusions: People living in the Kailash Sacred Landscape depend significantly on WNEPs, and this is especially critical in times of food shortage. The WNEPs have considerable potential as an important supplement to cultivated food crops. Farmers prioritise species with multiple use values and popular vegetables. However, there are numerous challenges and interventions needed to ensure conservation and management of species and their continued availability to support food security and local livelihoods.

Keywords: Wild and non-cultivated edible plants, Kailash Sacred Landscape, Traditional knowledge, Food security

\footnotetext{
* Correspondence: aryal.kamal@gmail.com; kamal.aryal@icimod.org

${ }^{1}$ Research Centre for Applied Science and Technology, Tribhuvan University,

Kritipur, Kathmandu, Nepal

${ }^{3}$ International Centre for Integrated Mountain Development (ICIMOD), GPO

Box 3226, Kathmandu, Nepal

Full list of author information is available at the end of the article
} 


\section{Background}

The majority of rural communities living in mountain and hill regions use wild and non-cultivated edible plant species (WNEPs) for food, medicine and other purposes [1-3]. WNEPs cover a wide range; they include wild fruit, nuts, leaves, roots, shoots and whole plants collected from forests, hedges and grassland; plants growing naturally alongside the actual crop in cultivated and fallow agricultural land; and plants established in the wild or in fields from seed that has dispersed from previously grown crops [4-9]. On occasion, plants that grow in the wild around some villages are collected as WNEPs and may be protected and managed in home gardens or agricultural fields in other villages where they count as crops.

Throughout the Himalayan region, WNEPs contribute substantially to food security, help maintain health and offer economic opportunities for millions of mountain people [10-12]. They are eaten in a myriad of ways-raw in salads and pickle, boiled in curries and soups, fried and steamed-depending on preference and taste [13, 14]. Many of these plants have cultural values, while some are considered sacred and used in religious and cultural events [11-13]. A number of studies in the Himalaya have documented WNEP species used as regular food [1, 2, 10-12] and shown that WNEPs play a significant role in fulfilling daily food requirements, especially in rural areas.

Notwithstanding the contribution to livelihoods and well-being, WNEPs have received little attention in the Himalayan region, with the exception to some extent of medicinal plants. There have been only a few studies of the diversity, use and local management practices of WNEPs $[2,11,13,15]$ and none on status and availability. Little is known about household consumption patterns or their role in household-level food and nutrition security and healthcare. Many studies have focussed simply on listing wild edible species and noting their use as food or medicine [1, 2, 9-13, 15, 16]. Furthermore, most research and development interventions under government programmes have paid little or no attention to this important sector [17-19]. Quantitative information on the presence, abundance, use and management of WNEPs is essential as a basis for developing effective conservation and management strategies that ensure that these species can continue to contribute to and, where possible, be used to improve food security.

The Kailash Sacred Landscape (KSL) is a transboundary landscape culturally linked to the region around Mount Kailash and shared by Nepal, India and the People's Republic of China. It is home to many ethnic communities and is a rich repository of WNEPs. Local people are known to rely heavily on these plant species for their livelihoods [20], but the actual availability, use, contribution to livelihoods and engagement of household members are poorly documented. The present study selected Khar Village Development Committee (VDC) in Darchula District in KSL Nepal to investigate the diversity of WNEPs, how each species is being used, the role in and implications for livelihoods and local perceptions on conservation and management differentiated by gender.

\section{Methods}

\section{Study site and people}

The study was conducted in all nine wards of Khar VDC of Darchula District in the Far Western Development Region of Nepal, located at 29.761128 to $29.817314 \mathrm{~N}$ latitude and 80.597531 to 80.683363 E longitude (Fig. 1). Khar VDC is a predominantly rural mid-hill area, with a total area of $26 \mathrm{~km}^{2}$ at an elevation of 1353-3236 masl. The vegetation is sub-tropical in the lower parts and temperate at higher elevation with mostly fragmented areas of deciduous, coniferous and mixed forest and areas of cultivated land along the hill slopes (mostly rain-fed terraces) and valley bottoms (mostly irrigated). Close to half of the VDC area (51\%) is covered by forest, $44 \%$ is agricultural land, $4 \%$ shrub land, $0.3 \%$ water bodies, $0.1 \%$ grassland and $0.07 \%$ settlement area [21]. The VDC is about a 3 -h walk from Khalanga Bazaar, the district headquarters of Darchula. It is also connected by a rural road to the bazaar (ca. $14 \mathrm{~km}$ ), but vehicular access is only possible during winter and spring.

In 2010, the VDC had a population of 4272 (2056 male, 2216 female) in 698 households [22]; the average household size of 7.1 is high compared to the national average of 4.9. The literacy rate is low (61\% of respondents were non-literate). The dominant castes are Chhetri and Brahmin with a few households of Dalits. The major castes in the village include Manyal, Sitoli, Dobal, Mahar, Tamata, Bisht, Dadal, Bohara and Thagunna.

\section{Research approach and methodology}

Figure 2 shows the research study framework. Three broad approaches were used with multiple tools. Quantitative and qualitative primary data were collected using a range of participatory tools followed by a household survey; the results were supplemented with secondary data obtained from a literature review.

\section{Participatory tools}

A range of participatory rural appraisal tools was used to gather a wide range of information. A total of $45 \mathrm{key}$ informants (18 female, 27 male) aged from 28 to 78 and representing all nine wards were interviewed individually. Key informants were selected at the village level with the help of the Api-Nampa VDC level conservation committee members, focussing on people expected to have extensive 


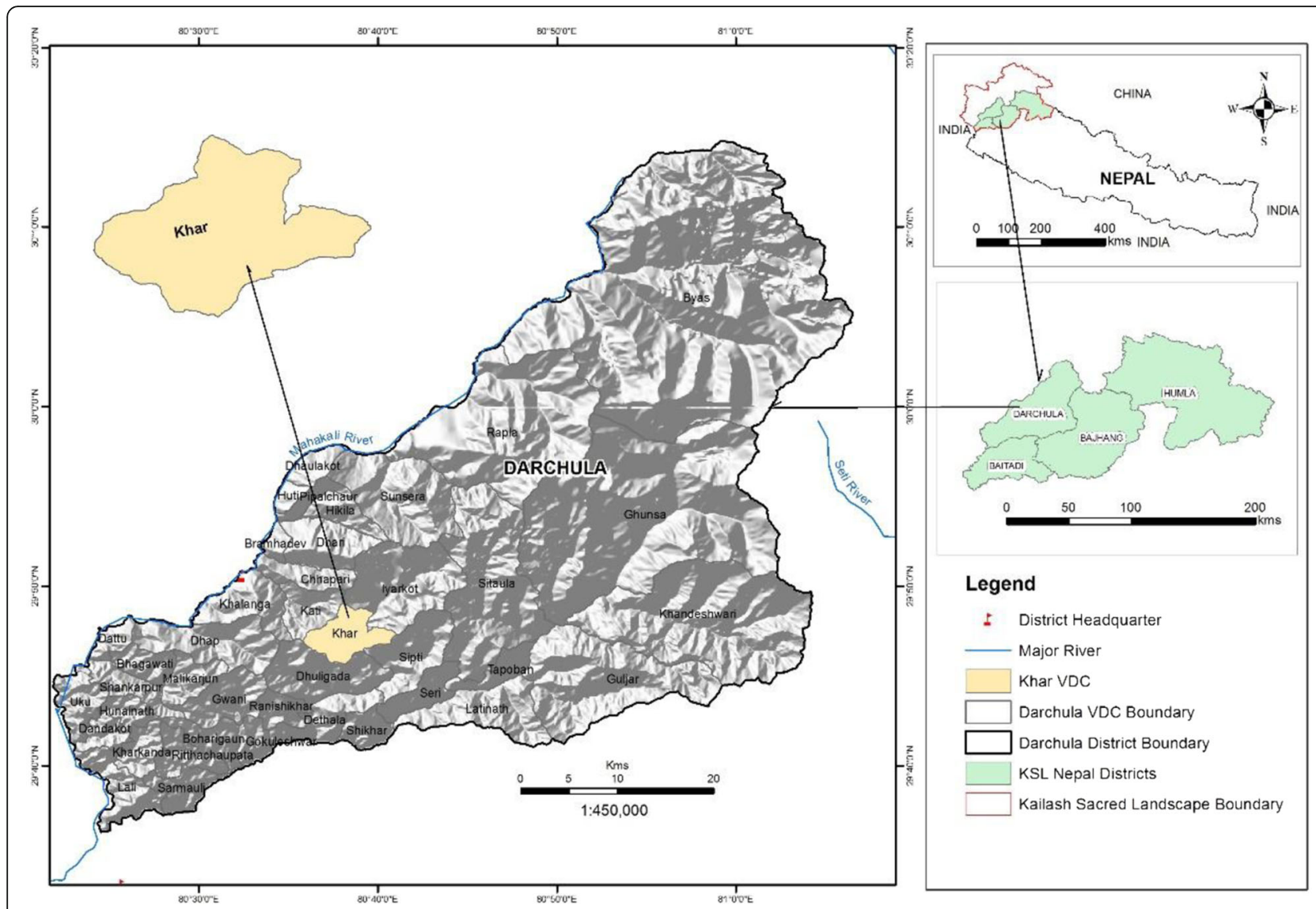

Fig. 1 The study site: Khar VDC in Darchula District in KSL Nepal

knowledge of WNEPs. Nine were specifically selected as local healers (one from each ward) who had been treating people for various health-related problems. Key informants were interviewed about their perceptions of the availability, uses and status of WNEPs and their contribution to local livelihoods.

Ten focus group discussions (one in each ward and one with representatives from the nine wards and other key institutions) were organised with 7-12 people in each group (82 participants: 40 women, 42 men). The discussions focussed on the general status and use of WNEPs in the VDC and local issues and initiatives on WNEP management.

Rapid market assessments were conducted at a local market (Dallekh Bazar) and the market at district headquarters (Khalanga Bazar) to identify the WNEPs available in different seasons, their market value and trends in use from the viewpoint of buyers and sellers.

A VDC level local crop diversity fair was organised in February 2015 to which farmers brought samples of all the WNEP species that they use that were available at the time. The aim was to make a rapid assessment of the general richness and status of non-cultivated plants available at that time. This fair provided a unique opportunity for individuals and community members to display their local plant material, as well as to share and document associated knowledge. During the fair, specimens of uncultivated plant species were collected and identified and herbarium were prepared.

WNEPs were also collected and identified in four field visits held in winter (February 2015), spring (May 2015), summer (July, 2016) and autumn (October 2016) by a multidisciplinary team consisting of a socio-economist, natural resources management expert, taxonomist and social mobiliser. Each field visit lasted for 15 days and covered all nine wards. The study team visited areas where species were found extensively in situ with the help of a social mobiliser and collected unidentified specimens for discussion with key informants. Information about species habitats was recorded, and photos were taken for future reference. Specimens were identified, and recent family and scientific names were assigned with the help of reference collections [23-27] and an expert taxonomist from the Central Department of Botany, Tribhuvan University.

\section{Household survey}

A detailed household survey with a structured questionnaire was used to obtain information about the use of 


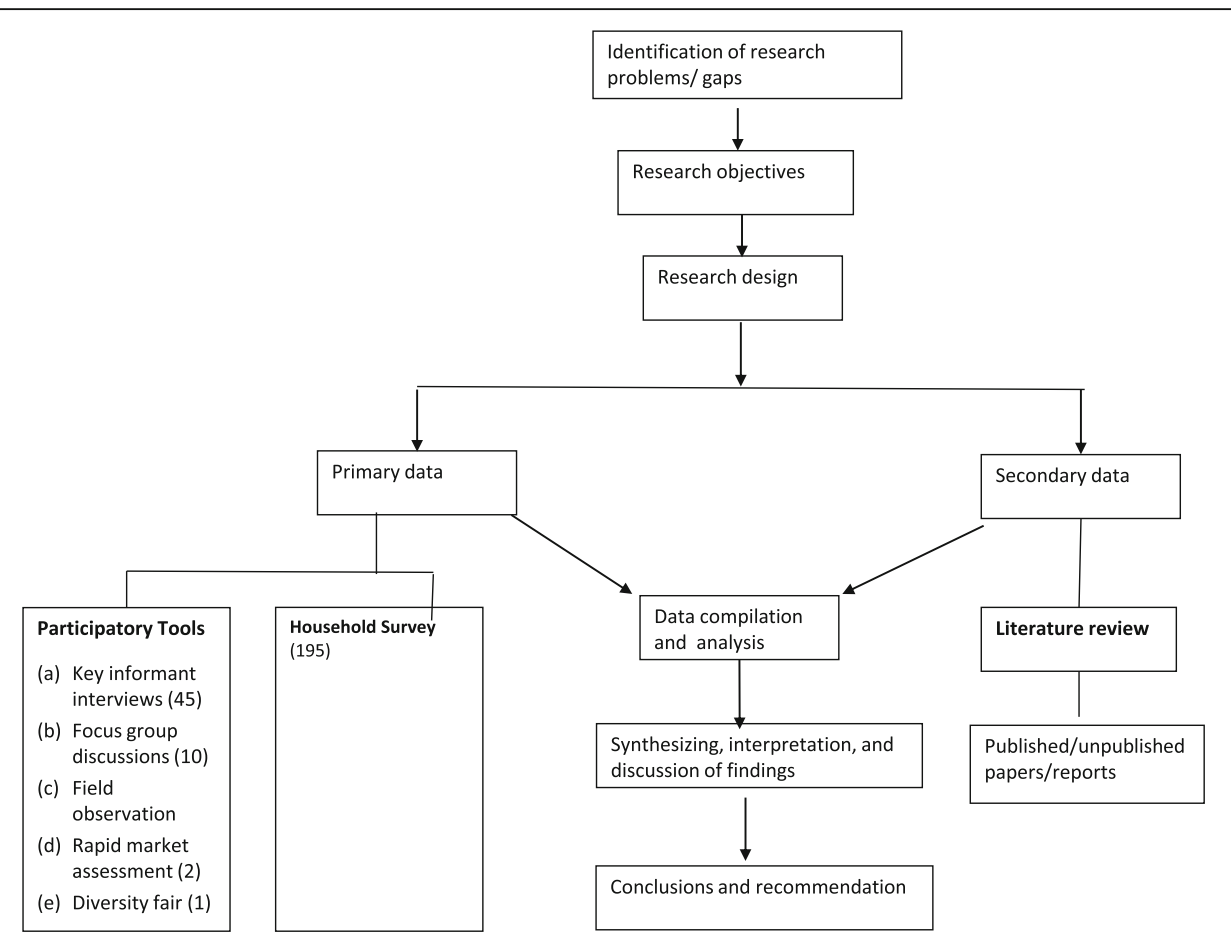

Fig. 2 Research study framework

WNEPs and any local-level management initiatives and the socio-economic and demographic features of the local population. The information gathered using the participatory tools was used in the survey design.

The sample size was determined using the following formula:

Number of households to be interviewed

$$
=\frac{Z_{1-\alpha}^{2} * N * P(1-P)}{\left(e^{2} * N\right)+\left(Z_{1-\alpha}^{2} * P(1-P)\right.}
$$

where $N$ is the total number of households $(N=698)$, $Z$ is the level of confidence (assumed value for $90 \%$ level of confidence is 1.65), $P$ is the estimate of the indicator to be measured (assumed value 50\% in the absence of any prior information) and $e$ is the margin of error to be attained (assumed level of precision is set at 5\%).

This gave a sample size of 195 households. In order to ensure proper representation from each ward, the sample was distributed proportionally according to the number of households in each ward. Within each ward, households were selected by random sampling with the help of computer-generated random numbers.

\section{Analytical tools}

Data was analysed using descriptive analysis and frequency calculation techniques, and results are presented in figures. In addition, informant consensus factor (ICF) was calculated to determine the homogeneity of the information and degree of overall agreement in using plant species with medicinal values-the species that are used for treating health-related problems at household level. The following formula was used [28]

$$
\mathrm{ICF}=\mathrm{Nur}-\mathrm{Nt} / \mathrm{Nur}-1
$$

Here, Nur is the number of use reports mentioned by the informant for the given species and $\mathrm{Nt}$ is the number of taxa (species) used by majority of the households.

Use value (UV) was calculated for individual plant species to give quantitative measures of its relative importance to the informants objectively [29]. Use value was calculated by using the following equation: $U V s=\Sigma U / n$, where UV refers to the use value of a species, $U$ is the number of use reports mentioned by the respondents and $n$ is the total number of respondents interviewed.

\section{Prior informed consent}

Before the study commenced, we shared the purpose and objectives with the community and relevant stakeholders in a half-day interactive meeting held in Dallekh village in Khar VDC. Prior informed consent was taken from the household respondents as well as all participants in the participatory interviews and discussions about the documentation and dissemination of local knowledge and use of WNEP species for study purposes. 


\section{Results}

Agriculture and food security

Agriculture was the major source of livelihoods for the majority of households (92\%); the major crops are maize, barley, wheat, finger millet and potato. However, only 5\% of households were able to meet all their annual food requirements from their own production; the remainder were only food sufficient for 10 months or less. Households adopted multiple coping strategies during the food deficit months to meet their food requirements, including seasonal migration for work to the district headquarters and various parts of India, sale of agricultural and livestock products, collection and selling of yarsagumba (Cordyceps sinensis) and collection of WNEPs.

\section{Diversity of WNEP species}

A total of 99 WNEPs belonging to 59 families were identified and documented (Table 1). They included 96 angiosperms, 1 gymnosperm and 2 pteridophytes, with 7 in the family Moraceae, 6 Rosaceae, 5 Urticaceae, 4 Polygonaceae and 3 each in Araceae, Dioscoreaceae, Amaranthaceae, Lamiaceae and Combretaceae. Herbs and trees were the most common life forms (Fig. 3).

\section{Diversity of use}

All households were using a range of different WNEPs for food, spice, medicinal and religious purposes. The most common uses were as food (fruit 40 species, vegetables 31 species), medicine (30 species), others (16 species) and spice (10 species). In a few cases, WNEPs formed the main meal for a short period (e.g. boiled Dioscorea spp.). Other uses included religious and traditional rituals, making pickles, ripening bananas, extracting cooking oil, washing and dyeing, and income generation; 35 species had multiple uses (Fig. 4, Table 1). The most commonly used parts were the fruit (45), leaves (31), and stems/shoots (17). Bark, buds, bulbs, flowers, tubers and corms, roots and seeds were also used (Table 1). Most uses (about 66\%) were specific to a particular plant part, although sometimes plant parts had multiple uses (e.g. as religious offerings and as medicine). In around two thirds of the species, only one plant part was used; in the others, multiple parts were used.

A total of 30 plant species have been used for household-level healthcare (Table 1). Diseases cured through the local knowledge system in the study sites were grouped into eight major types, and ICF was calculated for those diseases and health-related problems (Table 2). These include stomach disorder (diarrhoea/ dysentery), cuts and wounds, fever and headache, skin diseases/skin irritation, worms in stomach, nausea and vomiting, snake and scorpion bites and cough and cold. The values of ICF was found maximum for worms in the stomach (0.99) and minimum for skin disease treatment (0.67). Eight species were used to cure stomach disorder having maximum (178) number of use reports followed by cuts and wounds (160), and lowest use reports was found for skin disease (4) treatment (Table 2).

WNEPs used as vegetables for nutrition and food security In terms of regular food, one of the most important contributions of WNEPs was as a vegetable (Fig. 4). All respondents reported that they regularly used WNEPs as a vegetable. The most frequently collected species were Dioscorea bulbifera L., Dioscorea deltoidea Wall. ex Griseb., Urtica dioica L., Fagopyrum esculentum Moench, Dryopteris cochleata (D. Don) C. Chr. and Paeonia emodi Royle. Almost all respondents (92\%) used WNEPs to meet their daily vegetable requirements, with $75 \%$ depending exclusively on WNEPs for 1-3 months of the year and $10 \%$ for more than 3 months (Fig. 5).

The key perceptions of households on WNEPs and reasons for using them as vegetables are summarised in Fig. 6. The most common advantages of WNEPs were considered to be that they were tasty and nutritious $(85 \%)$ and also freely available (68\%).

\section{Annual extraction and use}

The estimated annual mean harvested weight of eight important species is shown in Fig. 7. The largest harvest was of $P$. emodi, a local seasonal vegetable locally known as heto found in the forest $(150 \mathrm{~kg})$, followed by F. esculentum and D. cochleata. Species like D. bulbifera (a tuber boiled as a vegetable) and $U$. dioica $\mathrm{L}$. are also important as sources of income as they can be sold in the local market. A few species have a significant local economic value, and people have started collecting and marketing some high-demand species like P. emodi, whose leaves are used to treat diarrhoea, and $D$. cochleata, an edible fern shoot which is even popular in big cities. Some 13\% of households sell these plants, earning an average of US $\$ 150$ per season. However, WNEPs are not a major source of cash income for most households.

The estimation of UV or relative importance of the frequently used vegetable species in the study site revealed that although the mean annual harvest of the species like Paeonia emodi and Fagopyrum esculentum is higher than Dryopteris cochleata (Fig. 7), the use value of Dryopteris cochleata (0.98) is higher than Paeonia emodi (0.96) and Fagopyrum esculentum (0.74). The use value (UV) of most important species used as vegetables in the study site is presented in Fig. 8.

\section{Gender roles in WNEP collection, utilisation, and management}

Respondents were asked who in the household did what related to WNEP use. Overall, the roles and responsibilities 
Table 1 Wild and non-cultivated edible plants identified in Khar VDC, Kailash Sacred Landscape, Nepal

\begin{tabular}{|c|c|c|c|c|c|c|c|c|c|}
\hline & Family & $\begin{array}{l}\text { Botanical } \\
\text { name }\end{array}$ & $\begin{array}{l}\text { English } \\
\text { name }\end{array}$ & $\begin{array}{l}\text { Nepali } \\
\text { name }\end{array}$ & $\begin{array}{l}\text { Local } \\
\text { name }\end{array}$ & Use $^{a}$ & $\begin{array}{l}\text { Parts } \\
\text { used }\end{array}$ & Remarks & $\begin{array}{l}\text { Specimen } \\
\text { number }\end{array}$ \\
\hline 1 & Acoraceae & $\begin{array}{l}\text { Acorus } \\
\text { calamus L. }\end{array}$ & $\begin{array}{l}\text { Flag } \\
\text { root, } \\
\text { myrtle flag }\end{array}$ & Bojho & Bojho & M & $R$ & $\begin{array}{l}\text { Dried rhizome } \\
\text { used to treat } \\
\text { sore throat, } \\
\text { coughs and } \\
\text { colds }\end{array}$ & D142 \\
\hline 2 & Adoxaceae & $\begin{array}{l}\text { Viburnum } \\
\text { erubescens } \\
\text { Wall. }\end{array}$ & & Bajrang & Ganaule & $\mathrm{F}$ & $\mathrm{F}$ & Fruit eaten & D305 \\
\hline 3 & Adoxaceae & $\begin{array}{l}\text { Viburnum } \\
\text { mullaha Buch.- } \\
\text { Ham.ex D. Don }\end{array}$ & & Kavase & Titmelau & $\mathrm{F}$ & $\mathrm{F}$ & $\begin{array}{l}\text { Fruit sour } \\
\text { but eaten }\end{array}$ & D278 \\
\hline 4 & Amaranthaceae & $\begin{array}{l}\text { Amaranthus } \\
\text { lividus L. }\end{array}$ & Amaranth & Marshi & Latte & v & L, Sh & $\begin{array}{l}\text { Leaves and } \\
\text { young shoots } \\
\text { eaten as a } \\
\text { green vegetable }\end{array}$ & D500 \\
\hline 5 & Amaranthaceae & $\begin{array}{l}\text { Amaranthus } \\
\text { spinosus L. }\end{array}$ & Amaranth & Marshi & $\begin{array}{l}\text { Kanya } \\
\text { marshi/ } \\
\text { chuwa }\end{array}$ & v & L, Sh & $\begin{array}{l}\text { Young leaves and } \\
\text { shoots eaten as a } \\
\text { green vegetable }\end{array}$ & D283 \\
\hline 6 & Amaranthaceae & $\begin{array}{l}\text { Amaranthus } \\
\text { viridis L. }\end{array}$ & Amaranth & Marshi & $\begin{array}{l}\text { Ghiya } \\
\text { marshi }\end{array}$ & $\mathrm{V}, \mathrm{O}$ & $\begin{array}{l}\text { L, Sh, } \\
\text { Se }\end{array}$ & $\begin{array}{l}\text { Young shoots and } \\
\text { leaves eaten as a } \\
\text { green vegetable; } \\
\text { seeds ground to } \\
\text { flour and used to } \\
\text { make chapattis; } \\
\text { seeds fried in } \\
\text { ghee and honey } \\
\text { and made into } \\
\text { round balls to } \\
\text { be eaten (ladoo/geda) }\end{array}$ & D316 \\
\hline 7 & Amaryllidaceae & $\begin{array}{l}\text { Allium } \\
\text { spp. }\end{array}$ & & & Dhunu & s & L & $\begin{array}{l}\text { Dried plant leaves } \\
\text { used in curries }\end{array}$ & D160 \\
\hline 8 & Amaryllidaceae & $\begin{array}{l}\text { Allium } \\
\text { wallichii Kunth }\end{array}$ & $\begin{array}{l}\text { Jimbur or } \\
\text { Himalayan } \\
\text { onion }\end{array}$ & Jimbu Jhar & $\begin{array}{l}\text { Sekkwa/ } \\
\text { sekuwa }\end{array}$ & s & W & $\begin{array}{l}\text { Dried plant used } \\
\text { in dal and curries }\end{array}$ & D50 \\
\hline 9 & Anacardiaceae & $\begin{array}{l}\text { Pistacia } \\
\text { chinensis } \\
\text { subsp. } \\
\text { integerrima } \\
\text { (J.L. Stewart ex Brandis) } \\
\text { Rech.f. }\end{array}$ & $\begin{array}{l}\text { Insect } \\
\text { gall in } \\
\text { Pistacia }\end{array}$ & Kakarsingee & Kakarsingee & M & Gall & $\begin{array}{l}\text { Gall used to treat } \\
\text { snake and } \\
\text { scorpion bites }\end{array}$ & D294 \\
\hline 10 & Apiaceae & Angelica archangelica $\mathrm{L}$. & & & Ganano & $S, M$ & $\mathrm{R}, \mathrm{Se}$ & $\begin{array}{l}\text { Root ground and } \\
\text { made into soup to } \\
\text { treat stomach pain. } \\
\text { Seeds ground to } \\
\text { flour and used } \\
\text { as spice in curry }\end{array}$ & D101 \\
\hline 11 & Araceae & $\begin{array}{l}\text { Arisaema } \\
\text { flavum } \\
\text { (Forssk.) } \\
\text { Schott }\end{array}$ & & Bako & Bako & v & T & $\begin{array}{l}\text { Corms (tubers) } \\
\text { boiled in ash } \\
\text { and salt to } \\
\text { remove toxic } \\
\text { elements, cleaned, } \\
\text { made into a } \\
\text { paste and } \\
\text { mixed with } \\
\text { buckwheat flour } \\
\text { to prepare curry }\end{array}$ & D196 \\
\hline 12 & Araceae & $\begin{array}{l}\text { Arisaema } \\
\text { tortuosum } \\
\text { (Wall.) } \\
\text { Schott }\end{array}$ & $\begin{array}{l}\text { Whipcord } \\
\text { cobra lily }\end{array}$ & Bako & Bako & v & T & $\begin{array}{l}\text { Boiled tubers } \\
\text { eaten as vegetable }\end{array}$ & D412 \\
\hline 13 & Araceae & $\begin{array}{l}\text { Colocasia } \\
\text { esculenta (L.) } \\
\text { Schott. }\end{array}$ & Taro & Pidaalu & Pidaalu & v & $R, S, L$ & $\begin{array}{l}\text { Rhizome boiled } \\
\text { and eaten as a } \\
\text { vegetable; young } \\
\text { stem and leaves } \\
\text { used as a vegetable } \\
\text { and in pickle }\end{array}$ & D119 \\
\hline 14 & Arecaceae & $\begin{array}{l}\text { Phoenix } \\
\text { humilis } \\
\text { Royle }\end{array}$ & & Thakal & Thakil/thakilo & $\mathrm{F}, \mathrm{O}$ & $F, S$ & $\begin{array}{l}\text { Fruit eaten; pith } \\
\text { from stem eaten; } \\
\text { stem used to } \\
\text { make thatched roofs }\end{array}$ & D284 \\
\hline 15 & Asparagaceae & & $\begin{array}{l}\text { Asaparagus, } \\
\text { wild Asparagus }\end{array}$ & Kurilo & Jhijhirkani & $\mathrm{V}, \mathrm{M}$ & R, Sh & $\begin{array}{l}\text { Shoots and leaves } \\
\text { eaten as a vegetable; }\end{array}$ & D140 \\
\hline
\end{tabular}


Table 1 Wild and non-cultivated edible plants identified in Khar VDC, Kailash Sacred Landscape, Nepal (Continued)

\begin{tabular}{|c|c|c|c|c|c|c|c|c|c|}
\hline & Family & $\begin{array}{l}\text { Botanical } \\
\text { name }\end{array}$ & $\begin{array}{l}\text { English } \\
\text { name }\end{array}$ & $\begin{array}{l}\text { Nepali } \\
\text { name }\end{array}$ & $\begin{array}{l}\text { Local } \\
\text { name }\end{array}$ & Use $\mathrm{a}^{\mathrm{a}}$ & $\begin{array}{l}\text { Parts } \\
\text { used }\end{array}$ & Remarks & $\begin{array}{l}\text { Specimen } \\
\text { number }\end{array}$ \\
\hline & & $\begin{array}{l}\text { Asparagus } \\
\text { racemosus } \\
\text { Willd. }\end{array}$ & & & & & & $\begin{array}{l}\text { roots used } \\
\text { to treat } \\
\text { urinary and } \\
\text { liver problems }\end{array}$ & \\
\hline 16 & Asteraceae & $\begin{array}{l}\text { Ageratina } \\
\text { adenophora } \\
\text { (Spreng.) R.M. } \\
\text { King \& H. Rob. }\end{array}$ & $\begin{array}{l}\text { Crofton } \\
\text { weed }\end{array}$ & Banmara & Banmara & M & L & $\begin{array}{l}\text { Juice from crushed } \\
\text { leaves used to } \\
\text { treat wounds } \\
\text { and cuts }\end{array}$ & \\
\hline 17 & Asteraceae & $\begin{array}{l}\text { Ageratum } \\
\text { conyzoides } \\
\text { (L.) L. }\end{array}$ & $\begin{array}{l}\text { Billygoat- } \\
\text { weed }\end{array}$ & Gandhe & Gandhe & M & L & $\begin{array}{l}\text { Leaves crushed } \\
\text { and juice used } \\
\text { to treat cuts } \\
\text { and wounds }\end{array}$ & D73 \\
\hline 18 & Asteraceae & $\begin{array}{l}\text { Artemisia } \\
\text { indica } \\
\text { Willd. }\end{array}$ & $\begin{array}{l}\text { Mug-wort, } \\
\text { Indian } \\
\text { worm } \\
\text { wood fleabane }\end{array}$ & Titepati & Kuljo & $R, M$ & L & $\begin{array}{l}\text { Leaves used in } \\
\text { death ceremonies; } \\
\text { leaves crushed and } \\
\text { juice used to treat } \\
\text { skin problems (irritation) }\end{array}$ & D506 \\
\hline 19 & Berberidaceae & $\begin{array}{l}\text { Berberis } \\
\text { aristata DC. }\end{array}$ & $\begin{array}{l}\text { Barberry/ } \\
\text { Nepal } \\
\text { Barberry/ } \\
\text { common } \\
\text { Barberry }\end{array}$ & Chutro & Chutro & $\begin{array}{l}\mathrm{F}_{\mathrm{M}} \mathrm{O} \\
\mathrm{M}\end{array}$ & $\mathrm{F}, \mathrm{Ba}$ & $\begin{array}{l}\text { Fruit eaten; bark } \\
\text { used as a dye } \\
\text { and to treat } \\
\text { diarrhoea, piles } \\
\text { and malaria }\end{array}$ & D190 \\
\hline 20 & Berberidaceae & $\begin{array}{l}\text { Berberis } \\
\text { asiatica } \\
\text { Roxb. ex DC. }\end{array}$ & $\begin{array}{l}\text { Barberry/ } \\
\text { Nepal } \\
\text { Barberry }\end{array}$ & Kirmando & Kirmada & $\mathrm{F}, \mathrm{O}$ & $\mathrm{F}, \mathrm{Ba}$ & $\begin{array}{l}\text { Fruit eaten; bark } \\
\text { used as a dye }\end{array}$ & D116 \\
\hline 21 & Bombacaceae & $\begin{array}{l}\text { Bombax } \\
\text { ceiba L. }\end{array}$ & $\begin{array}{l}\text { Silk cotton } \\
\text { tree, Simal } \\
\text { tree }\end{array}$ & Simal & Simal & v & $\mathrm{Fl}$ & $\begin{array}{l}\text { Flowers used in } \\
\text { a vegetable curry }\end{array}$ & D230 \\
\hline 22 & Cannabaceae & $\begin{array}{l}\text { Cannabis } \\
\text { sativa L. }\end{array}$ & $\begin{array}{l}\text { True hemp, } \\
\text { Indian hemp, } \\
\text { marijuana }\end{array}$ & Bhang & Bhango & $\mathrm{O}, \mathrm{M}$ & $\mathrm{Se}, \mathrm{L}$ & $\begin{array}{l}\text { Roasted seeds used } \\
\text { to make pickle or } \\
\text { eaten raw; green } \\
\text { leaves occasionally } \\
\text { used to make snacks } \\
\text { (pakauda); green leaves } \\
\text { made into a paste and } \\
\text { applied to the forehead } \\
\text { to treat high fever }\end{array}$ & D402 \\
\hline 23 & Chenopodiaceae & $\begin{array}{l}\text { Chenopodium } \\
\text { album L. }\end{array}$ & $\begin{array}{l}\text { Lamb's } \\
\text { quarter }\end{array}$ & $\begin{array}{l}\text { Bethe } \\
\text { sag }\end{array}$ & $\begin{array}{l}\text { Betu/ } \\
\text { charchare }\end{array}$ & v & L & $\begin{array}{l}\text { Leaves and young } \\
\text { shoots eaten as a } \\
\text { green vegetable }\end{array}$ & D229 \\
\hline 24 & Combretaceae & $\begin{array}{l}\text { Terminalia } \\
\text { bellirica } \\
\text { (Gaertn.) } \\
\text { Roxb. }\end{array}$ & $\begin{array}{l}\text { Belleric } \\
\text { myrobalan }\end{array}$ & Barro & Barado & $F, M$ & $\mathrm{Se}, \mathrm{F}$ & $\begin{array}{l}\text { Ripe fruit eaten; } \\
\text { seeds used to treat } \\
\text { coughs and colds }\end{array}$ & D100 \\
\hline 25 & Combretaceae & $\begin{array}{l}\text { Terminalia } \\
\text { chebula } \\
\text { Retz. }\end{array}$ & $\begin{array}{l}\text { Chebulie } \\
\text { myrobalan, } \\
\text { yellow } \\
\text { myrobalan }\end{array}$ & Harro & Harado & $F, M$ & $\mathrm{Se}, \mathrm{F}$ & $\begin{array}{l}\text { Fruit eaten; fruit } \\
\text { and seeds used to } \\
\text { treat coughs and colds }\end{array}$ & D154 \\
\hline 26 & Commelinaceae & $\begin{array}{l}\text { Commelina } \\
\text { benghalensis } \mathrm{L} .\end{array}$ & $\begin{array}{l}\text { Day } \\
\text { flower }\end{array}$ & $\begin{array}{l}\text { Kane } \\
\text { Sag }\end{array}$ & Kanya sag & v & L, Sh & $\begin{array}{l}\text { Young leaves and } \\
\text { shoots eaten as a } \\
\text { green vegetable }\end{array}$ & D131 \\
\hline 27 & Convolvulaceae & $\begin{array}{l}\text { Cuscuta } \\
\text { reflexa } \\
\text { Roxb. }\end{array}$ & Dodder & $\begin{array}{l}\text { Aakas } \\
\text { beli }\end{array}$ & Megh & M & w & $\begin{array}{l}\text { Whole plant used } \\
\text { to prepare medicine } \\
\text { to treat livestock } \\
\text { with cough and } \\
\text { throat allergy }\end{array}$ & D300 \\
\hline 28 & Cucurbitaceae & $\begin{array}{l}\text { Coccinia } \\
\text { grandis (L.) } \\
\text { Voigt }\end{array}$ & $\begin{array}{l}\text { Ivy gourd, } \\
\text { Kavai fruit }\end{array}$ & Golkakri & Golyakakadi & v & $\mathrm{F}$ & $\begin{array}{l}\text { Fruits eaten as } \\
\text { a vegetable }\end{array}$ & D280 \\
\hline 29 & Cucurbitaceae & $\begin{array}{l}\text { Momordica } \\
\text { dioica } \\
\text { Roxb. ex } \\
\text { Willd. }\end{array}$ & & Bankarela & Bankarela & v & $\mathrm{F}$ & $\begin{array}{l}\text { Immature fruit } \\
\text { eaten as a green } \\
\text { vegetable }\end{array}$ & D205 \\
\hline 30 & Dioscoreaceae & $\begin{array}{l}\text { Dioscorea } \\
\text { bulbifera L. }\end{array}$ & $\begin{array}{l}\text { Palmate } \\
\text { leaved yam }\end{array}$ & Githi & Githo & v & T & $\begin{array}{l}\text { Tubers boiled and } \\
\text { eaten as a vegetable }\end{array}$ & D429 \\
\hline 31 & Dioscoreaceae & $\begin{array}{l}\text { Dioscorea } \\
\text { deltoidea } \\
\text { Wall. ex } \\
\text { Griseb. }\end{array}$ & $\begin{array}{l}\text { Cush- } \\
\text { cush yam }\end{array}$ & Bhyakur & Bhyakur & V, & $B, T$ & $\begin{array}{l}\text { Bulbil and tubers } \\
\text { boiled and eaten } \\
\text { as a vegetable }\end{array}$ & D432 \\
\hline 32 & Dioscoreaceae & & & Ban tarul & Ban taud & $V, R$ & $B, T$ & & D438 \\
\hline
\end{tabular}


Table 1 Wild and non-cultivated edible plants identified in Khar VDC, Kailash Sacred Landscape, Nepal (Continued)

\begin{tabular}{|c|c|c|c|c|c|c|c|c|c|}
\hline & Family & $\begin{array}{l}\text { Botanical } \\
\text { name }\end{array}$ & $\begin{array}{l}\text { English } \\
\text { name }\end{array}$ & $\begin{array}{l}\text { Nepali } \\
\text { name }\end{array}$ & $\begin{array}{l}\text { Local } \\
\text { name }\end{array}$ & $U_{s e} e^{a}$ & $\begin{array}{l}\text { Parts } \\
\text { used }^{\text {b }}\end{array}$ & Remarks & $\begin{array}{l}\text { Specimen } \\
\text { number }\end{array}$ \\
\hline & & $\begin{array}{l}\text { Dioscorea } \\
\text { hamiltonii } \\
\text { Hook.f. }\end{array}$ & $\begin{array}{l}\text { Air potato, } \\
\text { potato yam }\end{array}$ & & & & & $\begin{array}{l}\text { Tubers and bulbils } \\
\text { cooked and eaten. } \\
\text { Boiled tubers are used } \\
\text { during religious event } \\
\text { first day of Nepali } \\
\text { Month Magh (January) }\end{array}$ & \\
\hline 33 & Dryopteridaceae & $\begin{array}{l}\text { Dryopteris } \\
\text { cochleata } \\
\text { (D. Don) } \\
\text { C. Chr. }\end{array}$ & $\begin{array}{l}\text { Edible } \\
\text { fern } \\
\text { shoot }\end{array}$ & Niuro & Liundo & $\mathrm{V}, \mathrm{O}$ & L, Sh & $\begin{array}{l}\text { Young coiled fronds } \\
\text { and shoots cooked } \\
\text { and eaten as a vegetable; } \\
\text { sold in urban markets } \\
\text { (high demand) }\end{array}$ & D113 \\
\hline 34 & Elaeagnaceae & $\begin{array}{l}\text { Elaeagnus } \\
\text { parvifolia Wall. } \\
\text { ex Royle }\end{array}$ & Oleaster & Kankoli & Guyaalo & $\mathrm{F}$ & $\mathrm{F}$ & Fruit eaten & D266 \\
\hline 35 & Ericaceae & $\begin{array}{l}\text { Rhododendron } \\
\text { arboreum Sm. }\end{array}$ & Rhododendron & Laligurans & Gurauns & $\mathrm{M}, \mathrm{O}$ & $\mathrm{Fl}$ & $\begin{array}{l}\text { Flowers eaten; nectar } \\
\text { used to treat diarrhoea } \\
\text { and dysentery }\end{array}$ & D218 \\
\hline 36 & Euphorbiaceae & $\begin{array}{l}\text { Phyllanthus } \\
\text { emblica L. }\end{array}$ & $\begin{array}{l}\text { Indian } \\
\text { gooseberry }\end{array}$ & Amala & Aaula & $F, M$ & $\mathrm{~F}$ & $\begin{array}{l}\text { Fruit eaten raw and dried; } \\
\text { fruit used in preparation of } \\
\text { some Ayurvedic medicines } \\
\text { for treating indigestion }\end{array}$ & D307 \\
\hline 37 & Fabaceae & $\begin{array}{l}\text { Albizia } \\
\text { procera } \\
\text { (Roxb.) } \\
\text { Benth. }\end{array}$ & $\begin{array}{l}\text { White } \\
\text { siris }\end{array}$ & Siris & Siris (not edible) & $\mathrm{O}$ & $\mathrm{L}$ & $\begin{array}{l}\text { Leaves used to cover } \\
\text { bananas to ripen them }\end{array}$ & D85 \\
\hline 38 & Fabaceae & $\begin{array}{l}\text { Bauhinia } \\
\text { variegata } \mathrm{L} .\end{array}$ & $\begin{array}{l}\text { Mountain } \\
\text { ebony, } \\
\text { White bauli }\end{array}$ & Koiralo & Koiral & $\mathrm{V}, \mathrm{M}$ & $\mathrm{Bu}, \mathrm{Fl}$ & $\begin{array}{l}\text { Buds and flowers used } \\
\text { as a vegetable and in } \\
\text { pickle; flowers used to } \\
\text { make soup to treat } \\
\text { bacillary dysentery }\end{array}$ & D236 \\
\hline 39 & Fagaceae & $\begin{array}{l}\text { Castanopsis } \\
\text { tribuloides (Sm.) } \\
\text { A.DC. }\end{array}$ & Chestnut & Katus & Katauj & $F, R$ & $\mathrm{~F}$ & $\begin{array}{l}\text { Fruit eaten and offered } \\
\text { to gods during rituals }\end{array}$ & D145 \\
\hline 40 & Fagaceae & $\begin{array}{l}\text { Quercus } \\
\text { lanata Sm. }\end{array}$ & $\begin{array}{l}\text { Woolly- } \\
\text { leaved oak }\end{array}$ & Baanjha & Baanjha & $\mathrm{F}$ & $\mathrm{F}$ & Fruit (lekaal) eaten & D480 \\
\hline 41 & Gentianaceae & $\begin{array}{l}\text { Swertia } \\
\text { chirayita } \\
\text { (Roxb. ex } \\
\text { Fleming) } \\
\text { Karsten }\end{array}$ & Chiretta & Chiraita & Chiraito & M & W & $\begin{array}{l}\text { Whole plant used to } \\
\text { treat fever, diabetes, } \\
\text { and skin diseases }\end{array}$ & D299 \\
\hline 42 & Hippocastanaceae & $\begin{array}{l}\text { Aesculus } \\
\text { indica } \\
\text { (Wall. ex } \\
\text { Cambess.) } \\
\text { Hook. }\end{array}$ & $\begin{array}{l}\text { Indian } \\
\text { horse } \\
\text { chestnut }\end{array}$ & Pangar & Pangar & $\mathrm{M}, \mathrm{O}$ & $\mathrm{F}$ & $\begin{array}{l}\text { Roasted fruit eaten to kill } \\
\text { stomach worms; fruit } \\
\text { used for washing clothes }\end{array}$ & D214 \\
\hline 43 & Juglandaceae & $\begin{array}{l}\text { Juglans } \\
\text { regia } \mathrm{L} \text {. }\end{array}$ & Walnut & Okhar & Okhad & $F, R$ & $\mathrm{~F}$ & $\begin{array}{l}\text { Fruit eaten and offered } \\
\text { to gods during festivals }\end{array}$ & D233 \\
\hline 44 & Lamiaceae & $\begin{array}{l}\text { Mentha } \\
\text { arvensis L. }\end{array}$ & Mint & Pudina & Padamchal & $S, M$ & $\mathrm{~L}$ & $\begin{array}{l}\text { Leaves used in pickle; } \\
\text { juice from leaves used } \\
\text { for cooling in summer }\end{array}$ & D110 \\
\hline 45 & Lamiaceae & $\begin{array}{l}\text { Mentha } \\
\text { spicata L. }\end{array}$ & Mint & Pudina & Padamchal & $S, M$ & L & $\begin{array}{l}\text { Leaves used as spice in } \\
\text { pickle; leaves used as } \\
\text { medicine to reduce 'body heat' }\end{array}$ & D248 \\
\hline 46 & Lamiaceae & $\begin{array}{l}\text { Perilla } \\
\text { frutescens } \\
\text { (L.) Britton }\end{array}$ & Perilla & Silame & Bhangiro & s & Se & $\begin{array}{l}\text { Seeds roasted and ground } \\
\text { to use in pickle }\end{array}$ & D387 \\
\hline 47 & Lardizabalaceae & $\begin{array}{l}\text { Holboellia } \\
\text { latifolia Wall. }\end{array}$ & & & Ghopala & $\mathrm{F}$ & $\mathrm{F}$ & Ripe fruit eaten & D493 \\
\hline 48 & Lauraceae & $\begin{array}{l}\text { Cinnamomum } \\
\text { glanduliferum } \\
\text { (Wall.) Meisn. }\end{array}$ & $\begin{array}{l}\text { Nepal } \\
\text { camphor } \\
\text { tree }\end{array}$ & Sunghandhaakokila & Sunghandhaakokila & $M, R$ & $\mathrm{Ba}, \mathrm{F}$ & $\begin{array}{l}\text { Bark and fruit } \\
\text { used to treat } \\
\text { coughs and colds, } \\
\text { toothache, and } \\
\text { swelling of muscles; } \\
\text { leaves and fruit offered } \\
\text { to gods during rituals }\end{array}$ & D96 \\
\hline 49 & Lauraceae & $\begin{array}{l}\text { Cinnamomum } \\
\text { tamala } \\
\text { (Buch.-Ham.) } \\
\text { T.Nees \& } \\
\text { Eberm. }\end{array}$ & $\begin{array}{l}\text { Bay } \\
\text { leaf }\end{array}$ & Tejpaat & Tejpaat/dalchini & s & L & $\begin{array}{l}\text { Dried leaves used as } \\
\text { spice for curries to } \\
\text { add flavour and smell }\end{array}$ & D82 \\
\hline
\end{tabular}


Table 1 Wild and non-cultivated edible plants identified in Khar VDC, Kailash Sacred Landscape, Nepal (Continued)

\begin{tabular}{|c|c|c|c|c|c|c|c|c|c|}
\hline & Family & $\begin{array}{l}\text { Botanical } \\
\text { name }\end{array}$ & $\begin{array}{l}\text { English } \\
\text { name }\end{array}$ & $\begin{array}{l}\text { Nepali } \\
\text { name }\end{array}$ & $\begin{array}{l}\text { Local } \\
\text { name }\end{array}$ & Use $^{a}$ & $\begin{array}{l}\text { Parts } \\
\text { used }^{b}\end{array}$ & Remarks & $\begin{array}{l}\text { Specimen } \\
\text { number }\end{array}$ \\
\hline 50 & Loranthaceae & $\begin{array}{l}\text { Loranthus } \\
\text { odoratus } \\
\text { Wall. }\end{array}$ & & Ajeru & Anjedu & $\mathrm{F}$ & $\mathrm{F}$ & Fruit very tasty & D178 \\
\hline 51 & Moraceae & $\begin{array}{l}\text { Ficus } \\
\text { auriculata } \\
\text { Lour. }\end{array}$ & $\begin{array}{l}\text { Eye's apron, } \\
\text { Moretan-bay } \\
\text { fig }\end{array}$ & Timilo & Timlo & $\mathrm{F}$ & $\mathrm{F}$ & Fruit eaten & D352 \\
\hline 52 & Moraceae & $\begin{array}{l}\text { Ficus } \\
\text { hispida L.f. }\end{array}$ & & Khasreto & Khasattya & $\mathrm{F}$ & $\mathrm{F}$ & Fruit eaten & D132 \\
\hline 53 & Moraceae & $\begin{array}{l}\text { Ficus } \\
\text { lacor } \\
\text { Buch.-Ham }\end{array}$ & & Kabhro & Kapado & v & $\mathrm{Bu}, \mathrm{Fl}$ & $\begin{array}{l}\text { Buds and flowers } \\
\text { boiled and eaten } \\
\text { as a vegetable } \\
\text { and pickle }\end{array}$ & D100 \\
\hline 54 & Moraceae & $\begin{array}{l}\text { Ficus } \\
\text { neriifolia } \\
\text { Sm. }\end{array}$ & & Dudhilo & Dudilo & $V, F$ & Sh, F & $\begin{array}{l}\text { Young shoots eaten } \\
\text { as a vegetable; } \\
\text { fruit eaten }\end{array}$ & D328 \\
\hline 55 & Moraceae & $\begin{array}{l}\text { Ficus } \\
\text { semicordata } \\
\text { Buch.-Ham. } \\
\text { ex Sm. }\end{array}$ & $\begin{array}{l}\text { Nepal } \\
\text { fodder } \\
\text { fig }\end{array}$ & Khaniyo & $\begin{array}{l}\text { Khannyo/ } \\
\text { khinne }\end{array}$ & $\mathrm{F}$ & $\mathrm{F}$ & Fruit eaten & D211 \\
\hline 56 & Moraceae & $\begin{array}{l}\text { Ficus subincisa } \\
\text { Buch.-Ham. } \\
\text { ex Sm. }\end{array}$ & & Berlo & $\begin{array}{l}\text { Belto/ } \\
\text { beldo }\end{array}$ & $\mathrm{F}$ & $\mathrm{F}$ & Ripe fruit eaten & D48 \\
\hline 57 & Moraceae & $\begin{array}{l}\text { Morus } \\
\text { serrata } \\
\text { Roxb. }\end{array}$ & Mulberry & Kimbu & Kimu & $\mathrm{F}, \mathrm{O}$ & $F, L$ & $\begin{array}{l}\text { Fruit eaten, } \\
\text { very popular } \\
\text { among children; } \\
\text { leaves used as } \\
\text { fodder, preferred } \\
\text { by goats }\end{array}$ & D333 \\
\hline 58 & Musaceae & $\begin{array}{l}\text { Musa } \\
\text { balbisiana } \\
\text { Colla }\end{array}$ & Banana & Bankera & Bankela & $F, R$ & $\mathrm{~F}$ & $\begin{array}{l}\text { Ripe fruit eaten } \\
\text { and offered to } \\
\text { gods during rituals }\end{array}$ & D127 \\
\hline 59 & Myricaceae & $\begin{array}{l}\text { Myrica } \\
\text { esculenta } \\
\text { Buch.-Ham. } \\
\text { ex D. Don }\end{array}$ & $\begin{array}{l}\text { Box } \\
\text { byrtle }\end{array}$ & Kafal & Kafal & $\mathrm{F}$ & $\mathrm{F}$ & $\begin{array}{l}\text { Fruit tasty } \\
\text { and popular }\end{array}$ & D318 \\
\hline 60 & Myrtaceae & $\begin{array}{l}\text { Syzygium } \\
\text { cumini } \\
\text { (L.) Skeels }\end{array}$ & $\begin{array}{l}\text { Black plum, } \\
\text { Java plum, } \\
\text { Indian black } \\
\text { berry }\end{array}$ & Jamun & Jamno & $\mathrm{F}$ & $\mathrm{F}$ & Fruit eaten & D246 \\
\hline 61 & Myrtaceae & $\begin{array}{l}\text { Syzygium } \\
\text { spp. }\end{array}$ & & & Phalda & $\mathrm{F}$ & $\mathrm{F}$ & Fruit eaten & D329 \\
\hline 62 & Nephrolepidaceae & $\begin{array}{l}\text { Nephrolepis } \\
\text { cordifolia } \\
\text { (L.) C. Presl }\end{array}$ & $\begin{array}{l}\text { Sword } \\
\text { fern }\end{array}$ & $\begin{array}{l}\text { Pani } \\
\text { amala }\end{array}$ & Rasmada & M & T & $\begin{array}{l}\text { Tubers eaten to } \\
\text { treat worms }\end{array}$ & D72 \\
\hline 63 & Oxalidaceae & $\begin{array}{l}\text { Oxalis } \\
\text { corniculata L. }\end{array}$ & $\begin{array}{l}\text { Indian } \\
\text { sorrel, } \\
\text { creeping } \\
\text { sorrel }\end{array}$ & $\begin{array}{l}\text { Chari } \\
\text { amilo }\end{array}$ & Chalmado & $\mathrm{s}$ & L & $\begin{array}{l}\text { Leaves used in } \\
\text { preparing pickle }\end{array}$ & D99 \\
\hline 64 & Paeoniaceae & $\begin{array}{l}\text { Paeonia } \\
\text { emodi } \\
\text { Royle }\end{array}$ & & & Hetto & v & L, Sh & $\begin{array}{l}\text { Young shoots and } \\
\text { leaves eaten as a } \\
\text { green vegetable, } \\
\text { fresh or sundried, } \\
\text { rehydrated, and } \\
\text { cooked (in winter) }\end{array}$ & D32 \\
\hline 65 & Phytolaccaceae & $\begin{array}{l}\text { Phytolacca } \\
\text { acinosa } \\
\text { Roxb. }\end{array}$ & & Jarko & Jarak/jarka & $\mathrm{V}, \mathrm{M}$ & $L, R$ & $\begin{array}{l}\text { Young leaves and } \\
\text { shoots eaten as a } \\
\text { green vegetable; } \\
\text { root used to treat } \\
\text { sickness after eating } \\
\text { buckwheat leaves }\end{array}$ & D4001 \\
\hline 66 & Pinaceae & $\begin{array}{l}\text { Pinus } \\
\text { roxburghii } \\
\text { Sarg. }\end{array}$ & $\begin{array}{l}\text { Chir pine, } \\
\text { Himalayan } \\
\text { long-leaved } \\
\text { pine }\end{array}$ & Salla & Sallo khote & M & La & $\begin{array}{l}\text { Resin used to clear } \\
\text { blood clots }\end{array}$ & D70 \\
\hline 67 & Poaceae & $\begin{array}{l}\text { Dendrocalamus } \\
\text { hamiltonii } \\
\text { Neer \& Arn. } \\
\text { ex Munro }\end{array}$ & $\begin{array}{l}\text { Tufted } \\
\text { bamboo }\end{array}$ & Bans & Bans & V & Sh & $\begin{array}{l}\text { Young shoots (tama) } \\
\text { eaten as a vegetable }\end{array}$ & D174 \\
\hline
\end{tabular}


Table 1 Wild and non-cultivated edible plants identified in Khar VDC, Kailash Sacred Landscape, Nepal (Continued)

\begin{tabular}{|c|c|c|c|c|c|c|c|c|c|}
\hline & Family & $\begin{array}{l}\text { Botanical } \\
\text { name }\end{array}$ & $\begin{array}{l}\text { English } \\
\text { name }\end{array}$ & $\begin{array}{l}\text { Nepali } \\
\text { name }\end{array}$ & $\begin{array}{l}\text { Local } \\
\text { name }\end{array}$ & Use $^{a}$ & $\begin{array}{l}\text { Parts } \\
\text { used }^{b}\end{array}$ & Remarks & $\begin{array}{l}\text { Specimen } \\
\text { number }\end{array}$ \\
\hline 68 & Poaceae & $\begin{array}{l}\text { Drepanostachyum } \\
\text { falcatum (Munro) } \\
\text { Keng f. }\end{array}$ & $\begin{array}{l}\text { Himalayan } \\
\text { Bamboo }\end{array}$ & Nigaalo & Nigaalo & $\mathrm{V}, \mathrm{O}$ & S, Sh & $\begin{array}{l}\text { Stem used to } \\
\text { make mats; } \\
\text { young shoots } \\
\text { eaten as a } \\
\text { vegetable }\end{array}$ & D290 \\
\hline 69 & Polygonaceae & $\begin{array}{l}\text { Fagopyrum } \\
\text { esculentum Moench }\end{array}$ & Buckwheat & Phapar & Phanpar & V & L, Sh & $\begin{array}{l}\text { Young shoots and } \\
\text { leaves eaten as a } \\
\text { vegetable }\end{array}$ & D443 \\
\hline 70 & Polygonaceae & $\begin{array}{l}\text { Fagopyrum } \\
\text { tataricum (L.) } \\
\text { Gaertn. }\end{array}$ & Buckwheat & Phapar & Phanpar & V & $L$ & $\begin{array}{l}\text { Young shoots } \\
\text { and leaves } \\
\text { eaten as a } \\
\text { vegetable }\end{array}$ & D205 \\
\hline 71 & Polygonaceae & $\begin{array}{l}\text { Polygonum } \\
\text { spp. }\end{array}$ & & & Halaudo & $S$ & $L$ & $\begin{array}{l}\text { Young leaves } \\
\text { used to } \\
\text { make pickle }\end{array}$ & D8 \\
\hline 72 & Polygonaceae & $\begin{array}{l}\text { Polygonum } \\
\text { verticillatum Birolli } \\
\text { ex Colla }\end{array}$ & & Nigali sag & Khinaudo & V & $\mathrm{L}$ & $\begin{array}{l}\text { Young leaves eaten } \\
\text { as a vegetable }\end{array}$ & D112 \\
\hline 73 & Ranunculaceae & $\begin{array}{l}\text { Aconitum } \\
\text { heterophyllum } \\
\text { Wall. ex Royle }\end{array}$ & Aconite & Atis & Atis & M & W, R & $\begin{array}{l}\text { Whole plant and roots } \\
\text { used to treat high fever } \\
\text { and abdominal pain }\end{array}$ & D260 \\
\hline 74 & Rosaceae & $\begin{array}{l}\text { Fragaria } \\
\text { nubicola } \\
\text { (Lindl.ex. } \\
\text { Hook.f) } \\
\text { Lacaita }\end{array}$ & & & $\begin{array}{l}\text { Gande } \\
\text { kafal }\end{array}$ & $\begin{array}{l}F, R \\
M\end{array}$ & $F, W$ & $\begin{array}{l}\text { Fruit eaten; whole plant } \\
\text { used in death rituals; } \\
\text { whole plant used to } \\
\text { treat stomach disorders }\end{array}$ & D68 \\
\hline 75 & Rosaceae & $\begin{array}{l}\text { Pyracantha } \\
\text { crenulata (Roxb. } \\
\text { ex D. Don) } \\
\text { M. Roem. }\end{array}$ & Fire horn & $\begin{array}{l}\text { Ghangyaru/ } \\
\text { kaatha gedi }\end{array}$ & Ghangyar & $\mathrm{F}$ & $\mathrm{F}$ & $\begin{array}{l}\text { Ripe fruit eaten in } \\
\text { large quantities }\end{array}$ & D108 \\
\hline 76 & Rosaceae & $\begin{array}{l}\text { Pyrus pashia } \\
\text { Buch.-Ham. } \\
\text { ex D. Don. }\end{array}$ & & Mayal & Mel & $\mathrm{F}$ & $\mathrm{F}$ & Fruit eaten & D239 \\
\hline 77 & Rosaceae & $\begin{array}{l}\text { Rubus } \\
\text { ellipticus } \\
\text { Sm. }\end{array}$ & $\begin{array}{l}\text { Golden } \\
\text { evergreen } \\
\text { raspberry }\end{array}$ & Ainselu & Anselu & $\mathrm{F}$ & $\mathrm{F}$ & $\begin{array}{l}\text { Fruit very } \\
\text { popular }\end{array}$ & D348 \\
\hline 78 & Rosaceae & $\begin{array}{l}\text { Rubus } \\
\text { foliolosus } \\
\text { D. Don }\end{array}$ & Rasberry & Ainselu & $\begin{array}{l}\text { Kalo } \\
\text { anselu }\end{array}$ & $F, R$ & $F, L, W$ & $\begin{array}{l}\text { Fruit eaten; } \\
\text { leaves or whole } \\
\text { plant used in } \\
\text { death ceremonies }\end{array}$ & D501 \\
\hline 79 & Rosaceae & $\begin{array}{l}\text { Rubus } \\
\text { niveus Thunb. }\end{array}$ & & Ainselu & $\begin{array}{l}\text { Katrya } \\
\text { anselu }\end{array}$ & $\mathrm{F}$ & $\mathrm{F}$ & Fruit eaten & D98 \\
\hline 80 & Rubiaceae & $\begin{array}{l}\text { Rubia manjith } \\
\text { Roxb. ex } \\
\text { Fleming }\end{array}$ & Indian madder & Majitho & Majitho & M & $S, L$ & $\begin{array}{l}\text { Stem and } \\
\text { leaves used } \\
\text { to treat cuts } \\
\text { and wounds }\end{array}$ & D103 \\
\hline 81 & Rutaceae & $\begin{array}{l}\text { Aegle } \\
\text { marmelos } \\
\text { (L.) Correa }\end{array}$ & Bael fruit & Bel & Bel & $F, R$ & $F, L$ & $\begin{array}{l}\text { Fruit pulp eaten; } \\
\text { leaves used } \\
\text { for religious } \\
\text { purposes, } \\
\text { especially offering } \\
\text { to gods during } \\
\text { rituals }\end{array}$ & D187 \\
\hline 82 & Rutaceae & $\begin{array}{l}\text { Zanthoxylum } \\
\text { armatum DC. }\end{array}$ & $\begin{array}{l}\text { Nepal } \\
\text { pepper, } \\
\text { prickly ash }\end{array}$ & Timur & Timur & $S, M$ & $\mathrm{~F}$ & $\begin{array}{l}\text { Fruit dried and } \\
\text { used as a } \\
\text { spice in pickles } \\
\text { and curries; } \\
\text { dried fruit used } \\
\text { in various allopathic } \\
\text { medicines like } \\
\text { indigestion and } \\
\text { nausea }\end{array}$ & D234 \\
\hline 83 & Sapindaceae & $\begin{array}{l}\text { Sapindus } \\
\text { mukorossi } \\
\text { Gaertn. }\end{array}$ & $\begin{array}{l}\text { Soap } \\
\text { nut }\end{array}$ & Reetha & $\begin{array}{l}\text { Reetha } \\
\text { (not edible) }\end{array}$ & $\mathrm{O}$ & $\mathrm{F}$ & $\begin{array}{l}\text { Fruit pulp } \\
\text { used to } \\
\text { wash hair }\end{array}$ & D431 \\
\hline 84 & Sapotaceae & $\begin{array}{l}\text { Diploknema } \\
\text { butyracea } \\
\text { (Roxb.) } \\
\text { H.J.Lam }\end{array}$ & $\begin{array}{l}\text { Nepal } \\
\text { butter fruit Phulwara }\end{array}$ & Chiuri & Chyuro & $\mathrm{O}, \mathrm{F}$ & $\begin{array}{l}\mathrm{Fl}, \mathrm{F} \\
\mathrm{Se}\end{array}$ & $\begin{array}{l}\text { Nectar from } \\
\text { flowers and } \\
\text { ripened fruit } \\
\text { (bhina) eaten; } \\
\text { seeds used to } \\
\text { make a butter }\end{array}$ & D268 \\
\hline
\end{tabular}


Table 1 Wild and non-cultivated edible plants identified in Khar VDC, Kailash Sacred Landscape, Nepal (Continued)

\begin{tabular}{|c|c|c|c|c|c|c|c|c|c|}
\hline & Family & $\begin{array}{l}\text { Botanical } \\
\text { name }\end{array}$ & $\begin{array}{l}\text { English } \\
\text { name }\end{array}$ & $\begin{array}{l}\text { Nepali } \\
\text { name }\end{array}$ & $\begin{array}{l}\text { Local } \\
\text { name }\end{array}$ & Use $^{a}$ & $\begin{array}{l}\text { Parts } \\
\text { used }^{b}\end{array}$ & Remarks & $\begin{array}{l}\text { Specimen } \\
\text { number }\end{array}$ \\
\hline & & & & & & & & $\begin{array}{l}\text { for cooking } \\
\text { vegetables } \\
\text { and others }\end{array}$ & \\
\hline 85 & Saurauiaceae & $\begin{array}{l}\text { Saurauia } \\
\text { napaulensis DC. }\end{array}$ & & Gogan & Gogan & $\mathrm{F}$ & $\mathrm{F}$ & $\begin{array}{l}\text { Fruit } \\
\text { eaten }\end{array}$ & D15 \\
\hline 86 & Saxifragaceae & $\begin{array}{l}\text { Bergenia ciliata } \\
\text { (Haw.) } \\
\text { Sternb. }\end{array}$ & Rock foil & Pakhanbed & $\begin{array}{l}\text { Pakhanbed/simpari } \\
\text { phool }\end{array}$ & M & $R$ & $\begin{array}{l}\text { Rhizome used to } \\
\text { make medicine to } \\
\text { treat kidney stones }\end{array}$ & D134 \\
\hline 87 & Schisandraceae & $\begin{array}{l}\text { Schisandra } \\
\text { grandiflora } \\
\text { (Wall.) Hookf. \& } \\
\text { Thomson }\end{array}$ & $\begin{array}{l}\text { Magnolia } \\
\text { Vine }\end{array}$ & & Haliyude & $\mathrm{F}$ & $\mathrm{F}$ & $\begin{array}{l}\text { Ripe } \\
\text { fruit eaten }\end{array}$ & D245 \\
\hline 88 & Smilacaceae & Smilax aspera L. & Green briers & Kukurdaino & Kukuldaino & $F, V$ & Sh, F & $\begin{array}{l}\text { Fruit eaten; } \\
\text { young } \\
\text { shoots eaten } \\
\text { as a vegetable }\end{array}$ & D218 \\
\hline 89 & Smilacaceae & $\begin{array}{l}\text { Smilax ovalifolia Roxb. ex D. } \\
\text { Don }\end{array}$ & Green briers & Kukurdaino & Kukuldaino & $F, V$ & Sh, F & $\begin{array}{l}\text { Fruit eaten; } \\
\text { young shoots } \\
\text { eaten as a vegetable }\end{array}$ & D68 \\
\hline 90 & Solanaceae & $\begin{array}{l}\text { Solanum } \\
\text { nigrum L. }\end{array}$ & & Kalokamai & Ninauni & $\mathrm{F}$ & $\mathrm{F}$ & Fruit eaten & D149 \\
\hline 91 & Trilliaceae & $\begin{array}{l}\text { Paris } \\
\text { polyphylla } \\
\text { Sm. }\end{array}$ & & Satuwa & Satuwa & $\mathrm{V}, \mathrm{M}$ & $L, R$ & $\begin{array}{l}\text { Tender leaves } \\
\text { eaten as a } \\
\text { vegetable; root } \\
\text { made into paste } \\
\text { and applied to } \\
\text { snake bite to } \\
\text { control the poison }\end{array}$ & D179 \\
\hline 92 & Urticaceae & $\begin{array}{l}\text { Boehmeria } \\
\text { rugulosa } \\
\text { Wedd. }\end{array}$ & & Getha & Githi & 0 & $\mathrm{Ba}$ & $\begin{array}{l}\text { Bark paste/powder } \\
\text { mixed with rice } \\
\text { flour to prepare } \\
\text { sel roti(a form of } \\
\text { rice doughnut); } \\
\text { bark paste used } \\
\text { as soda and } \\
\text { to wash clothes }\end{array}$ & D22 \\
\hline 93 & Urticaceae & $\begin{array}{l}\text { Debregeasia s } \\
\text { alicifolia (D. Don) } \\
\text { Rendle }\end{array}$ & & Tusaare & Tusaaro & $\mathrm{F}$ & $\mathrm{F}$ & Fruit eaten & D55 \\
\hline 94 & Urticaceae & $\begin{array}{l}\text { Girardinia } \\
\text { diversifolia } \\
\text { (Link) Friis }\end{array}$ & $\begin{array}{l}\text { Himalayan } \\
\text { Nettle }\end{array}$ & Allo Sisnu & Allo & $\mathrm{V}, \mathrm{O}$ & $\begin{array}{l}\text { L, Sh, } \\
\text { S, }\end{array}$ & $\begin{array}{l}\text { Young leaves } \\
\text { and shoots } \\
\text { eaten; fibre } \\
\text { extracted f } \\
\text { rom stems } \\
\text { used to make } \\
\text { clothes } \\
\text { and bags }\end{array}$ & D17 \\
\hline 95 & Urticaceae & $\begin{array}{l}\text { Gonostegia } \\
\text { hirta } \\
\text { (Blume ex Hassk.) } \\
\text { Miq. }\end{array}$ & & & Attinno & 0 & R & $\begin{array}{l}\text { Ground root } \\
\text { used to prepare } \\
\text { chapatti; ground } \\
\text { root used for } \\
\text { washing hair }\end{array}$ & D458 \\
\hline 96 & Urticaceae & $\begin{array}{l}\text { Urtica } \\
\text { dioica L. }\end{array}$ & $\begin{array}{l}\text { Stinging } \\
\text { nettle }\end{array}$ & Sisnu & Sisnu & $\mathrm{v}$ & L, Sh & $\begin{array}{l}\text { Young leaves } \\
\text { and shoots } \\
\text { used as a } \\
\text { vegetable }\end{array}$ & D16 \\
\hline 97 & Verbenaceae & $\begin{array}{l}\text { Callicarpa } \\
\text { arborea Roxb. }\end{array}$ & Beauty berry & Guyalo & Gwailo & $\mathrm{F}$ & $\mathrm{F}$ & Tasty fruit & D67 \\
\hline 98 & Violaceae & Viola L. & & & Juke jhaar & M & $L, R$ & $\begin{array}{l}\text { Leaves and roots used } \\
\text { to treat worms in children }\end{array}$ & D481 \\
\hline 99 & Vitaceae & Tetrastigma spp. & & & Pudaayen & $\mathrm{F}$ & $\mathrm{F}$ & Fruit eaten & D344 \\
\hline
\end{tabular}

ase: $F$ fruit, $V$ vegetable, $M$ medicine, $O$ other, $R$ religious, $S$ spice

${ }^{b}$ Part of plant used: $W$ whole plant, $B$ bulb, Ba bark, Bu buds, $F$ fruit, $F I$ flowers, La latex, $L$ leaf, $O$ other, $R$ root/rhizome, $S$ stem, Se seeds, Sh shoots, $T$ tuber/corm

in activities and decision-making on collection, processing, food preparation, storage and marketing of WNEPs were shared between men and women (Table 3). Irrespective of gender, about half of the respondents (49\%) stated that decisions and activities related to collection and harvesting were shared by men and women, with the remainder divided almost equally between women or men. Responsibility for processing was generally thought to be shared 


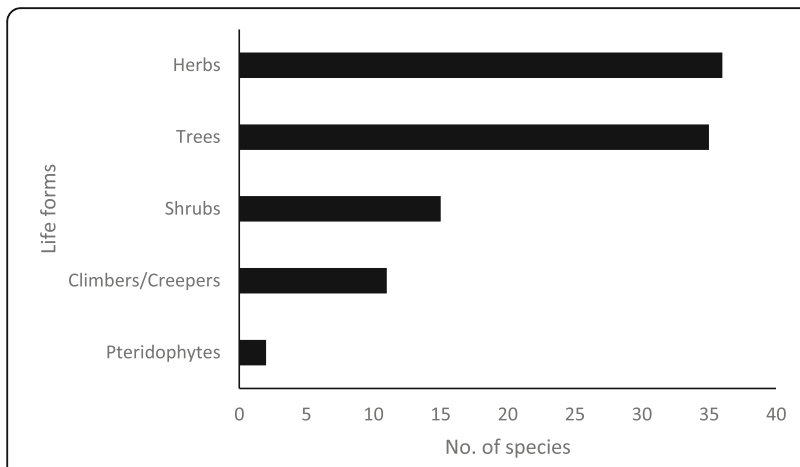

Fig. 3 Frequency of different life forms of WNEPs

equally (around 80\%) as was responsibility for conservation and management. However, women had much greater responsibility for preparation and storage.

\section{Local perceptions}

Respondents were asked about the existing and potential issues of concern related to WNEPs. The primary issues identified were premature and unsustainable harvesting (147), inadequate labour resources within the family (134) and time taken for collection (120) (Fig. 9). Other issues included neglect of local food, availability of ready-made food and problems identifying whether species are edible, especially among young collectors. We discussed these issues further in the FGDs. Of the ten FGDs (82 participants), eight groups also thought that the major issues for utilisation and management were lack of human resources due to migration for seasonal work, unsustainable harvesting and changing human lifestyles and taste. We also asked about current management practices. In all ten groups, participants mentioned in situ conservation of important species by almost all people in the village, with domestication of important species as the second most important strategy. This perception was supported by the data from the household survey. The great majority of respondents (86\%) reported practising in situ conservation and

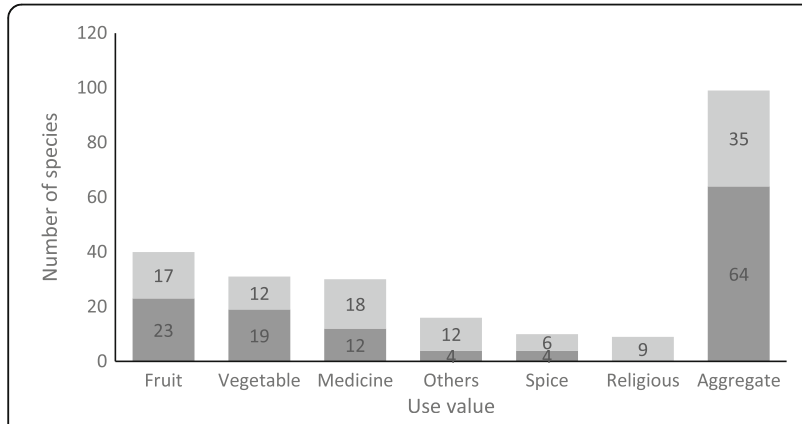

Fig. 4 Uses of WNEPs (single use, black-shaded; multiple use, grey-shaded)
Table 2 Categories of ailments and informant consensus factor (ICF)

\begin{tabular}{llll}
\hline Use categories & $\begin{array}{l}\text { No. of } \\
\text { taxa }\end{array}$ & $\begin{array}{l}\text { No. of use } \\
\text { reports }\end{array}$ & $\begin{array}{l}\text { Consensus } \\
\text { factor }\end{array}$ \\
\hline $\begin{array}{l}\text { Stomach disorder (diarrhoea/ } \\
\text { dysentry) }\end{array}$ & 8 & 178 & 0.96 \\
Cuts and wounds & 4 & 160 & 0.98 \\
Fever and headache & 6 & 125 & 0.96 \\
Skin diseases/skin irritation & 2 & 4 & 0.67 \\
Worms in stomach & 2 & 120 & 0.99 \\
Nausea and vomiting & 2 & 73 & 0.98 \\
Snake and scorpion bites & 2 & 8 & 0.85 \\
Cough and cold & 4 & 186 & 0.98 \\
\hline
\end{tabular}

domestication (38\%) of key species in their home gardens and agricultural fields.

\section{Discussion}

\section{Diversity of WNEPs and their use}

It is estimated that at least a billion people use WNEPs in their diet [30]. Millions of people in the Himalayan region depend on WNEPs for their daily food and vegetable requirements as well as for fresh fruit and medicines [30-33]. Our study documented 99 WNEP species currently used in various forms by the local people in the Kailash Sacred Landscape area in far west Nepal. A number of studies by other authors have documented a diverse range of WNEP species and uses in different parts of the Himalayan region, but most have not assessed status and availability, household consumption patterns or local management practices. The study in $\mathrm{Ti}$ betan community of China documented the use of 54 species for household consumption [34]. Similar study conducted in Tibetan communities of Nepal, China and India also documented 75 wild food plants of diverse uses at household level [35]. Singh et al. [36] documented 111 WNEPs used in Bandipora district in Kashmir, while other authors identified 112 WNEPs in

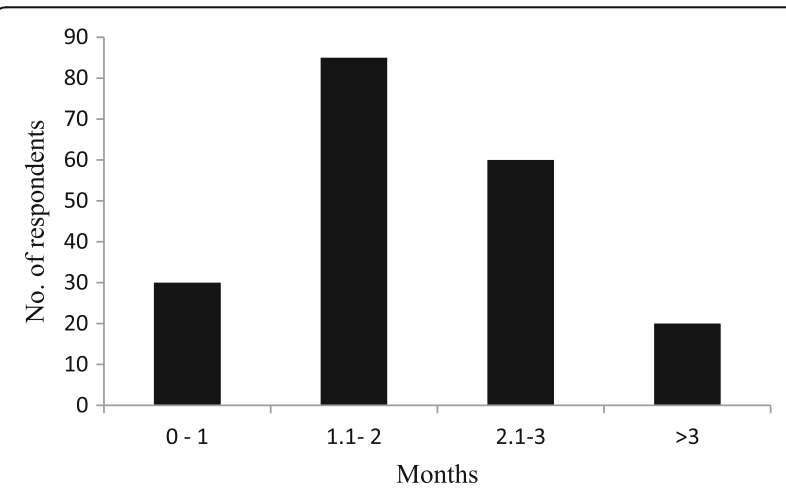

Fig. 5 Dependence on WNEPs on daily vegetable requirements $(N-195)$ 


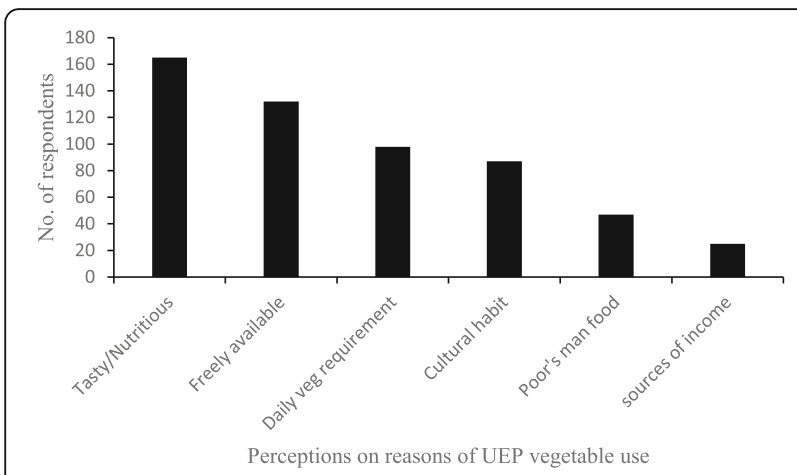

Fig. 6 Perceptions of and reasons for using WNEPs $(N=195)$

Dhading and Kaski districts in Nepal [16] and 62 in Bhutan [37]. Khan et al. [38] conducted assessment of wild edible plants of Sewa catchment area in Northwest Himalaya of India and listed 97 plant species used by local inhabitants for various uses. More than 380 nontimber forest products (NTFPs) were identified in Meghalaya in North East India [39] and 739 in the Kangchenjunga Landscape (India, Nepal and Bhutan) [40] of which many were WNEPs, although these were not separately listed.

WNEPs contributed substantially to the food requirements of the households in the study area. People preferred to collect species with multiple use value, but they also collected large quantities of species used purely as a vegetable. P. emodi, $U$. dioica, F. esculentum and $D$. cochleata were particularly popular and constituted an important source of vegetables in household food. A large quantity of $P$. emodi and F. esculentum is harvested, but the use value of $D$. cochleata was found higher, which might be attributable to their widespread distribution and abundance of the later species across the study area making them the first choice for collection and consumption. The average annual extraction of species used as vegetables was very high; this has also been observed by others. For example, in Dhusa VDC in Dhading district, Nepal, individual households were

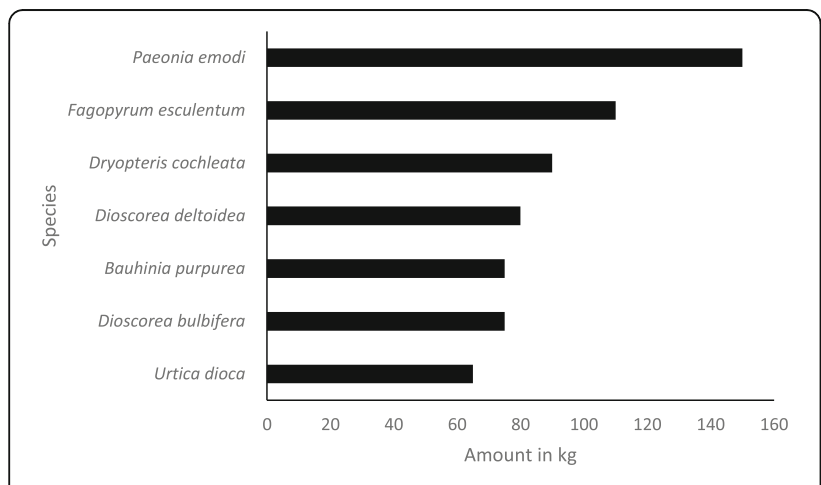

Fig. 7 Average extraction per annum of major WNEPs (in $\mathrm{kg}$ )

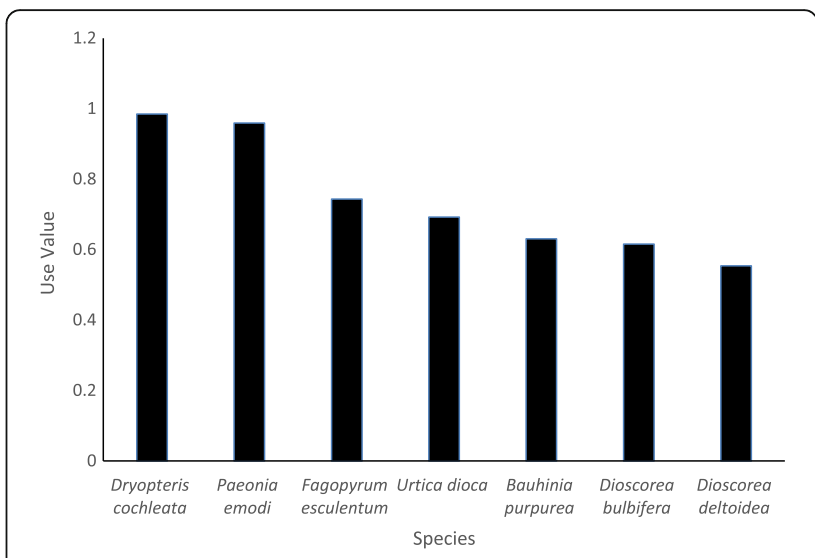

Fig. 8 Use value of frequently used vegetable species

observed to collect an average $200 \mathrm{~kg}$ of D. bulbifera per annum [13], while Chepang households in Gorkha district of Nepal consumed an average $364 \mathrm{~kg}$ of $D$. bulbifera and $96 \mathrm{~kg}$ of $U$. dioica per annum [41]. A diverse range of Dioscorea spp. is widely used and consumed by the local community in Himanchal and Similipal Biosphere Reserve in India [31, 42]. Together, these figures suggest that people are harvesting at least some WNEPs in large quantities, which has also been observed in studies conducted in other parts of the world [1, 2, 11, 15, 31, 32, 43-51]. Most people at the study site depended on WNEPs to fill their vegetable requirements for between 1 and 3 months or more a year. A study carried out among the Chepang people in Nepal reported that $58 \%$ of households depended on WNEPs for

Table 3 Division of responsibility for WNEP activities and decisions among men and women

\begin{tabular}{llll}
\hline Role and responsibility & \multicolumn{1}{l}{$N=195$} & & \\
\cline { 2 - 4 } & Women & Men & Both \\
\hline Activities & & & \\
Harvesting/collection & $55(28)$ & $45(23)$ & $95(49)$ \\
Processing & $25(13)$ & $20(10)$ & $150(77)$ \\
Preparation & $165(85)$ & $10(5)$ & $20(10)$ \\
Storage & $135(69)$ & $10(5)$ & $50(26)$ \\
Marketing/exchange & $75(38)$ & $37(19)$ & $83(43)$ \\
Conservation and management & $20(10)$ & $35(18)$ & $140(72)$ \\
Decision-making & & & \\
Harvesting/collection & $45(23)$ & $55(28)$ & $95(49)$ \\
Processing & $25(13)$ & $12(6)$ & $158(81)$ \\
Preparation & $185(95)$ & $5(3)$ & $5(3)$ \\
Storage & $160(82)$ & $10(5)$ & $25(13)$ \\
Marketing/exchange & $45(23)$ & $85(44)$ & $65(33)$ \\
Conservation and management & $48(25)$ & $30(15)$ & $117(60)$ \\
\hline
\end{tabular}

Note: figures in brackets are percentage of respondents 


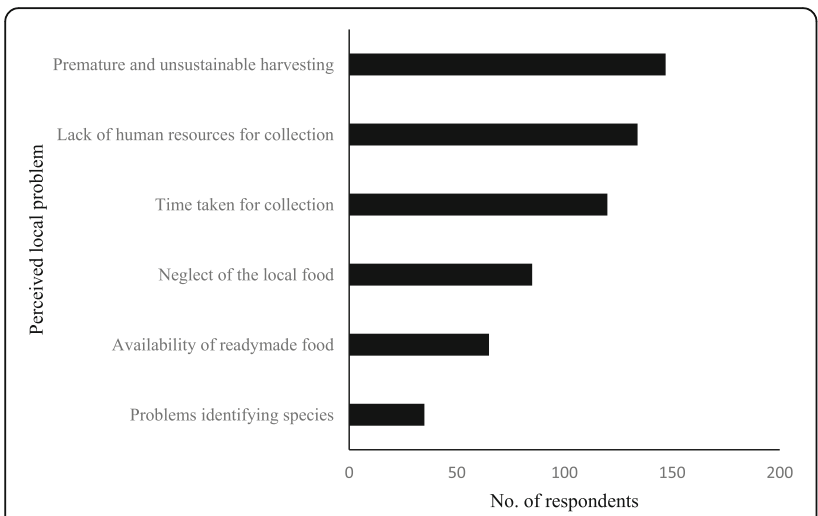

Fig. 9 Primary issues related to utilisation and management of WNEPS $(N=195)$

vegetables for up to 5 months a year [2], and in one village in India, people ate WNEPs as vegetables for at least 50-80 days per year [47]. A study in Burkina Faso showed $20 \%$ of all food items to come from wild/noncultivated sources [43], while non-cultivated greens are one of the major sources of vegetables in rural areas of Vietnam, eaten by almost all households [43]. Wild leafy vegetables are an important part of diet of people of Shiri in Daghestan, and 70\% of them are used as snacks. They are important in maintaining social life as the dried vegetables are sent as gifts to distant relatives and people visiting them at their place [52]. So, the wild vegetables are also culturally associated with the indigenous communities.

The studies highlight the importance of WNEPs in local diets but also indicate that the current trends in harvesting of some species may not be sustainable and could affect species availability in the future $[1,2,4,53]$.

WNEPs are considered to be an important source of vitamins and minerals [32, 54-56] and to contribute to energy and micronutrients for farm families throughout the year $[43,57]$. The study conducted in Naxi community of China depicted that wild edible plants play a very important role in safeguarding food and nutritional security [58]. This is also supported by other two studies conducted in India $[59,60]$. However, the precise nutritional composition of most of these foods is not known [61], although one study showed, for example, that the root crop from Dioscorea spp. contains five times more protein and fibre than potato and sweet potato [62]. Similarly, little is known about the actual contribution of WNEPs to people's daily food requirements, and this remains poorly studied. In addition to contributing to food and nutritional security, a wide range of WNEPs contribute to health and well-being as medicinal plants [4, $10,40,44,49,63-67]$. For example, most diseases in far west Nepal are treated by individuals and local healers using traditionally handed-down ethno-medicinal knowledge of plants, which have been protected and have flourished where ethnic traditions and beliefs are still strong $[44,68,69]$. The informant consensus factors for the medicinal plant use suggest that a number of plant species have been used for treating various ailments such as stomach disorder, colds and cough, wounds and cut, skin diseases, fever/headache, nausea and vomiting, worms in the stomach and snake and scorpion bites. Rural people, particularly in remote villages, have been using these plant species for generations to treat different diseases based on their indigenous knowledge. Similar treatments of various diseases were also documented in the other studies from the region [70-72]. Especially, local healers know how to prepare drugs from raw herbs through personal experience and ancestral prescription. Such drugs are regularly used and have proven to be effective, inexpensive and beneficial and with few side effects compared to allopathic drugs $[2,4,10,73]$. The use of herbs by traditional health practitioners is based on trust gained over generations and religious connections to such practices [4]. However, the use of plants as medicines is declining $[69,74]$, partly because there are fewer traditional healers due to lack of knowledge transfer. The younger generation has little interest in studying traditional forms of medicine.

Although WNEPs make a significant contribution to the livelihoods of local people in the more remote mountain regions, these species are less used in the daily diets of households in other areas $[2,11,13$, 15]. WNEPs have the potential to play an important role in maintaining and improving food security in the many rural areas where food security remains a cause for concern and in supplementing nutritionally poor diets that are otherwise low in vitamins and minerals. However, changing food habits, taste, and lifestyles and availability of ready-made foods in the market are contributing to an increasing neglect of traditional foods in rural diets. Collection and use of WNEPs is considered risky and time-consuming, and young people are becoming less familiar with WNEP species and forest environments and less able to identify suitable species for harvesting. Little is known about the sustainability of harvesting practices $[1,2$, $6,9,13,16,40]$, and reduced availability is also cited by various studies as one of the underlying causes of the declining use of WNEPs $[1-3,10-16]$. The use of WNEPs is likely to decrease further, threatening the retention of knowledge about this important component of livelihoods, culture and tradition [11, 13]. At the same time, sustainable use and management of these resources remain a prime concern for the millions of mountain people whose lives still depend on them [49], as well as being essential to ensure the basis for further exploitation of their potential. 


\section{Conservation and management of WNEPs}

The true status of WNEPs, their contribution to livelihoods and the interrelationship with other species in the region has yet to be studied systematically [33, 36, 44]. Recent and past studies remain inadequate as they have focussed more on compiling lists of species and less on analysing their contribution to nutrition and food security $[40,42]$. Despite their important contribution to nutrition, WNEPs have also received little attention in government food and nutrition programmes in the region $[2,33,44]$.

A number of studies have noted the decreasing availability of WNEPs $[2,15]$. The loss of WNEPs has many causes, including habitat degradation, rapid urbanisation and over exploitation, as well as changes in food habits $[75,76]$. Changes in agricultural practices towards increased monocropping, use of herbicides and pesticides and increased mechanisation and changes in forestry practices towards more managed regimes and plantation may all play a role. At the same time, some WNEP species are becoming more heavily exploited as urbanised populations become motivated to eat local products and farmers collect plants for sale in urban markets rather than personal consumption [15, 33, 44, 47, 74, 77-80]. Species with high use value are subjected to higher extraction, which may be unsustainable. Control of overexploitation and illegal harvesting will be essential to ensure sustainable management. A coordinated effort is needed from all sectors to develop and implement in situ conservation, domestication and other conservation and management strategies for long-term management of WNEP species [1-5, 13, 19, 31, 33]. Furthermore, WNEPs can be promoted through the large-scale cultivation by integrating them into agricultural systems and making markets profitable for the benefit of the people $[59,60]$ With the participation of local people and a wide range of other stakeholders, it will be possible to craft more holistic and culturally appropriate strategies for utilisation and management of WNEPs in the Western Himalayas [67].

Maintenance and use of WNEPs in the Kailash region, as in Nepal overall, is not just important for botanical studies or as an ecological exercise. The conservation and wise utilisation of the indigenous knowledge of useful plants can help in the improvement of living standard of poor people of Nepal. It equally holds true for several developing countries where similar ecological and socio-cultural landscapes exist [81]. These plants play a significant role in meeting the daily food requirements of thousands of people living in rural villages like Khar, and play an important part in their survival strategies $[1,2,31,37,45,82]$. WNEPs are not only important in times of famine or stress [74], they are an essential part of a mineral rich normal diet for millions of people [83-85]. WNEPs are important resources, and further study is essential to provide updated inventories and information about their availability and use. Local people must be involved in conservation and management, as they are both the guardians and users of the resources and have the greatest knowledge about them. It is also important to organise local-level WNEP fairs and local food festivals to raise awareness about the importance of WNEP species, revive interest among the younger generation, and motivate communities towards proactive management of these resources. Domestication of WNEPs where possible will be needed to ensure continued availability; thus, it would be beneficial to encourage cultivation and/or domestication of plants used for food, fodder, medicine and other purposes. Technical and material support will be very much needed in the initial stages. Domestication in home gardens would be a good starting point, as they offer increased availability of water, a mostly organic-based production system, easier protection against predators and close monitoring by the household members.

\section{Conclusions}

A total of 99 WNEPs species with high diversity and multiple use values were documented in the KSL Nepal. These plants play a significant role in household-level food and nutrition as well as health security. The local livelihood system depends heavily on traditions and values that are rooted in nature. WNEP species, now often used most heavily in times of food shortage, have the potential to become important alternatives to the usual food crops cultivated by farmers. Farmers gave priority to those species that provide them with a multitude of benefits such as food and nutritional security as well as household-level healthcare but also harvested large amounts of species popular as vegetables. Species like $P$. emodi, $D$ bulbifera L., $D$. deltoidea, $U$ dioica, and $F$. esculentum are an important part of local peoples' livelihoods. However, there is a growing pressure on such species, which suggests that there is an urgent need for conservation and management, which requires proper research and policy advocacy. These wild and noncultivated resources are crucial to local peoples' traditions and contribute strongly to subsistence. It is important to consider how such species can contribute to future food security. This requires an understanding of how to manage the cultural changes affecting the use of WNEPs and how to ensure sustainable availability. Integrated research and development programmes are urgently needed to address the issue.

Abbreviations

FGDs: Focus group discussions; KSL: Kailash Sacred Landscape; VDC: Village Development Committee; WNEP: Wild and non-cultivated edible plant species 


\section{Acknowledgements}

The authors wish to thank all the respondents and community members at the study sites who patiently shared their time, insights and views about WNEPs. The study was carried out under the Kailash Sacred Landscape Conservation and Development Initiative (KSLCDI) of the International Centre for Integrated Mountain Development (ICIMOD). The authors would like to thank Mr. Bhumiraj Upadhyay, warden of Api-Nampa Conservation Area, and his team for their cooperation and logistic support while conducting the survey. We also acknowledge the guidance and support of the Ministry of Forest and Soil Conservation, Government of Nepal, for implementing KSLCDI. The authors would like to thank ICIMOD and RECAST of Tribhuvan University for providing technical and managerial support to facilitate the research. We wish to thank Ms. Sabarnee Tuladhar from ICIMOD for her help in designing the questionnaire and Ms. Beatrice Murray from the UK for the language editing of the manuscript. We would like to thank the Department for International Development (DFID)-UK Aid, German Federal Ministry of Economic Cooperation and Development and German International Cooperation (GIZ) for providing financial support for the KSLCDI. Last but not least, this study was partially supported by core funds from ICIMOD contributed by the Governments of Afghanistan, Australia, Austria, Bangladesh, Bhutan, China, India, Myanmar, Nepal, Norway, Pakistan, Switzerland and the UK.

\section{Funding}

The funding support for this study came from the Department for International Development (DFID)-UK Aid, German Federal Ministry of Economic Cooperation and Development, and German International Cooperation (GIZ) under the Kailash Sacred Landscape Conservation and Development Initiative (KSLCDI) programme coordinated by International Centre for Integrated Mountain Development (ICIMOD). Partial funding came from ICIMOD's core fund contributed by different member countries.

\section{Availability of data and materials}

Availability of the structured and organised version of the data is available from the first author upon request. The voucher specimens are kept at Central Department of Botany at Tribhuvan University, Nepal, and will be available upon request.

\section{Disclaimer}

The views and interpretations in this publication are those of the authors and not necessarily attributable to their organisations.

\section{Authors' contributions}

$K A, S P, R C P, N C, R C$ and WN designed the study. KA and SP carried out the literature review and did the field study and data collection. KA, SP, RCP, NC, PC and WN analysed the data and wrote the manuscript. All authors approved the final version of the manuscript.

\section{Ethics approval and consent to participate}

A prior informed consent was taken from the farmers and local communities in the study sites. The study was conducted in Api-Nampa Conservation Area of Department of National Parks and Wild life Conservation of Government of Nepal, and prior approval and consent was taken from the government for this study.

\section{Consent for publication}

The manuscript does not include the individuals' photographs and images, videos, reprints and personal information; hence, no need of submitting the consent for publication.

\section{Competing interests}

The authors declare that they have no competing interests.

\section{Publisher's Note}

Springer Nature remains neutral with regard to jurisdictional claims in published maps and institutional affiliations.

\section{Author details}

${ }^{1}$ Research Centre for Applied Science and Technology, Tribhuvan University, Kritipur, Kathmandu, Nepal. ${ }^{2}$ Ecological Services Centre, Bharatpur, Chitwan, Nepal. ${ }^{3}$ International Centre for Integrated Mountain Development (ICIMOD),
GPO Box 3226, Kathmandu, Nepal. ${ }^{4}$ Agriculture and Forestry University, Rampur, Chitwan, Nepal.

Received: 27 September 2017 Accepted: 13 January 2018

Published online: 29 January 2018

\section{References}

1. Dorji Y. Women's roles in wild yam, conservation, management and use in Bhutan. In: Khadka M, Verma R, editors. Gender and biodiversity management in the Greater Himalayas, ICIMOD; 2012. p. 25-7.

2. Aryal KP, Berg A, Ogle BM. Uncultivated plants and livelihood support: a case study from the Chepang people of Nepal. Ethnobot Res Appl. 2009; 7:409-22.

3. Hawksworth DL. Human exploitation of biodiversity and conservation: a question of balance? In: Hawksworth DL, Bull AT, editors. Human exploitation and biodiversity and conservation, vol. 15. Dordrecht: Springer; 2006. p. 2341-2.

4. Rijal A. Surviving knowledge: ethnobotany of Chepang community from mid hills of Nepal. Ethbot Res Appl. 2011;9:181-215.

5. Modi M, Modi AT, Hendriks S. Potential role for wild vegetables in household food security: a preliminary case study in Kwazulu-Natal, South Africa. Afr J Food Agric Nutr. 2006;6(1):1-13.

6. Somnasang $\mathrm{P}$, Rathakette $\mathrm{P}$, Rathanapanya $\mathrm{S}$. The role of natural foods in Northeast Thailand. In: Lovelace GW, Subhadira S, Simaraks S, editors. Rapid rural appraisal in Northeast Thailand. Case studies. Khon Kaen: Khon Kaen University; 1988. p. 78-103.

7. FAO: Use and potential of wild plants in farm households. 1999. http:// www.fao.org/3/a-w8801e.HTM. Accessed 21 Feb 2017.

8. Hladik A, Dounias E. Wild yams of the American rain forest as potential food resources. In: Hladik CM, Hladik A, Linares OE, Pagezy H, Semple A, Hadley M, editors. Tropical forests, people and food. Biocultural interactions and applications to development. New York and Paris: UNESCO and the Parthenon Publishing Group; 1993. p. 163-76.

9. Kar A, Bora D, Borthakur SK, Goswami NK, Saharia D. Wild edible plant resources used by the Mizos of Mizoram, India. Kath Uni J Sci Eng Tech. 2013:9(1):106-26.

10. Adnan M, Begum S, Khan AL, Tareen AM, Lee IJ. Medicinal plants and their uses in selected temperate zones of Pakistani Hindu-Kush Himalaya. J Med Plant Res. 2012;6(24):4113-27.

11. Akhtar F. Uncultivated food in the context of poor people's livelihood: a Nayakrishi experience. In: Johnston MM, editor. Proceedings of the regional workshop on uncultivated foods and biodiversity, September 24 to 26 , Kathmandu, Nepal; 2001.

12. Yesudas BS. Conservation of uncultivated foods by local communities. LEISA India, 6(1). Valuing Crop Biodiversity. 2004. http://vikaspedia.in/agriculture/ best-practices/sustainable-agriculture/biodiversity/conservation-ofuncultivated-foods-by-local-communities. Accessed 15 Nov 2015.

13. Aryal KP. Wild and uncultivated plants. Options for livelihood support of the people in the middle hills of Nepal. Master's Thesis No. 31. Uppsala 2007, ISSN: 1653-834X, Swedish University of Agricultural Sciences (SLU), Uppsala, Sweden. 2007. https://www.slu.se/globalassets/ew/org/centrb/cbm/ dokument/publikationer-cbm/masteruppsatser/kamal-prasad-aryal-thesis.pdf. Accessed on 28 June 2016.

14. Pieroni A, Nebel S, Sanroro RF, Heinrich M. Food for two seasons: culinary uses of non-cultivated local vegetables and mushrooms in a south Italian village. Int J Food Sci Nutr. 2005;56(4):245-72.

15. Piya L, Maharjan KL, Joshi NP. Forest and food security of indigenous people: a case of Chepangs in Nepal. J Int Dev Coop. 2011;17(1):113-35.

16. Regmi BR, Aryal KP, Tamang BB, Shrestha PK: Homegardens: an opportunity to minimize pressure on slash and burn system and options for improving dietary diversity of Chepang households. In: Gautam R, Sthapit BR, Shrestha PK, editors. Enhancing the contribution of home gardens to on-farm management of plant genetic resources and to improve the livelihoods of Nepalese farmers: lessons learned and policy implications. Proceedings of home garden workshop, 6-7 August 2004, Pokhara, Nepal. LI-BIRD, Bioversity International and SDC. 2006. p. 35-47.

17. Kumar A, Pandey VC, Tewari DD, Singh AG. Traditional uses of medicinal plants for dermatological healthcare management practices by the Tharu tribal community of Utter Pradesh. India Gen Res Crop Eval. 2013;60:203-24. 
18. Aryal KP, Kerkhoff EE, Maskey N, Sherchan R. Shifting cultivation in sacred Himalayan landscape. A case study from Kangchenjunga Conservation Area. 1st ed. Kathmandu: WWF Nepal; 2010.

19. Shrestha SR. The state of uncultivated foods in Nepal biodiversity and uncultivated food plants. In: Johnston MM, editor. The proceedings of the regional workshop on uncultivated foods and biodiversity, September 24 to 26, Kathmandu, Nepal. USC Canada-Nepal; 2001.

20. Zomer R, Oli KP, editors. Kailash sacred landscape conservation initiative-feasibility assessment report. Kathmandu: ICIMOD; 2011.

21. ICIMOD. Feasibility study report and participatory natural resources management plan: Khar Village development committee in Api-Nampa Conservation Area, Darchula Nepal [Unpublished report]. Kathmandu: International Center for Integrated Mountain Development (ICIMOD); 2013.

22. CBS. Nepal living standard survey 2010/11, volume one. Kathmandu: Central Bureau of Statistics, Government of Nepal; 2011.

23. Watson MF, Mkiyama S, Ikeda H, Pendry C, Rajbhandari KR, Shrestha KK, editors. Flora of Nepal, vol. 3. Edinburgh: The Royal Botanic Garden; 2011.

24. Press JR, Shrestha KK, Sutton DA. Annotated checklist of the flowering plants of Nepal. London: The Natural History Museum; 2000.

25. Stainton JDA. Flowers of the Himalaya. A supplement. New Delhi: Oxford University Press; 1988.

26. The Plant List. Version 1.1. 2013. http://www.theplantlist.org/. Accessed 4 Nov 2017.

27. The Angiosperm Phylogeny Group (APG IV). An update of the angiosperm phylogeny group classification for the orders and families of flowering plants: APG IV. Bot J Linn Soc. 2016;181:1-20.

28. Trotter RT, Logan MH. Informant consensus. A new approach for identifying potentially effective medicinal plants. In: Etkin NL, editor. Plants in indigenous medicine and diet Rdgrave. New York: Bedford Hills; 1986.

29. Phillips O, Gentry AH, Reynel C, Wilkin P, Galvez-Durand BC. Quantitative ethnobotany and Amazonian conservation. Conserv Biol. 1994;8:225-48.

30. Burlingame B. Wild nutrition. J Food Compos Anal. 2000;13(2):99-100.

31. Sundriyal M, Sundryal RC. Underutilized edible plants of the Sikkim Himalaya: need for domestication. Curr Sci. 2003:85(6):731-6.

32. Sundriyal M, Sundryal RC. Wild edible plants of the Sikkim Himalaya: nutritive values of selected species. Econ Bot. 2001;55(3):377-90.

33. Aryal K, Kotru R, Phuntsho K. Unlocking uncultivated food for mountain livelihood: case from Hindu Kush, Himalayas. J Agric Environ. 2013;14:160-70.

34. Kang J, Kang Y, Ji X, Guo Q, Jacques G, Pietras M, Łuczaj L, Li D, Łuczaj L. Wild food plants and fungi used in the mycophilous Tibetan community of Zhagana (Tewo County, Gansu, China). J Ethnobiol Ethnomed. 2016;12:21.

35. Boesi A. Traditional knowledge of wild food plants in a few Tibetan communities. J Ethnobiol Ethnomed. 2014;10:75.

36. Singh B, Sultan P, Hassan QP, Gairola S, Bedi YS. Ethnobotany, traditional knowledge and diversity of wild edible plants and fungi: a case study in the Bandipora District of Kashmir Himalaya, India. J Herbs Spices Med Plants. 2016; https://doi.org/10.1080/10496475.2016.1193833.

37. Thapa $L$. The research project on edible wild plants of Bhutan and their associated traditional knowledge. J Fac Agr Shinshu Univ. 2009;45(1):43-8.

38. Khan M, Kumar S, Hamal IA, Koul S. Wild edible plants of Sewa catchment area in Northwest Himalaya. J Plant Devel Sci. 2009:1(1\&2):1-7.

39. Tiwari BK: Traditional management of NTFP/MAPs in north East India: a case study of bayleaf and broom grass. Paper presented at a consultation meeting on improving livelihoods of mountain communities through sustainable utilization of non-timber forest products at ICIMOD, 18-20 December 2003, Kathmandu, Nepal. 2003

40. Uprety Y, Poudel RC, Gurung J, Chettri N, Chaudhary RP. Traditional use and management of NTFPs in Kangchenjunga landscape; implications for conservation and livelihoods. J Ethnobiol Ethnomed. 2016:12:19.

41. Kafle G, Limbu P, Pradhan B, Jing F. Piloting ecohealth approach for addressing land use transition, climate change and human health issues. NGO Group Bulletin on Climate Change. Pokhara: Local Initiatives for Biodiversity, Research and Development (LI- BIRD); 2009. http://www. forestrynepal.org/images/publications/Kafle_ecohealth-climate-change.pdf. Accessed 20 Feb 2016

42. Kumar S, Das G, Shin H-S, Patra JK. Dioscorea spp. (a wild edible tuber): a study on its ethnopharmacological potential and traditional use by the local people of Similipal Biosphere Reserve, India. Front Pharmacol. 2017;8:52. https://doi.org/10.3389/fphar.2017.00052.

43. Ogle BM. Wild vegetables and micronutrient nutrition: studies on the significance of wild vegetables in women's diet in Vietnam. Comprehensive summaries of Uppsala Dissertations from the faculty of Medicine 1056. Uppsala: Acta Universitatis Upsaliensis; 2001. p. 5-59. ISBN 91-554-5068-7.

44. Uprety Y, Poudel RC, Shrestha KK, Rajbhandary S, Tiwari NN, Shrestha UB, Asselin H. Diversity of use and local knowledge of wild edible plant resources in Nepal. J Ethnobiol Ethnomed. 2012;8(1):16.

45. Talukdar T. Women's as key managers of biodiversity in shifting cultivation in Bangladesh. In: Khadka M, Verma M, editors. Gender and biodiversity management in the Greater Himalayas, Kathmandu, International Centre for Integrated Mountain Development; 2012. p. 21-4.

46. Tiwari JK, Ballabha R, Tiwari P. Some promising wild edible plants of Srinagar and its adjacent area in Alaknada valley of Garhwal Himalaya, India. J Am Sci. 2010;6(4):167-74.

47. Reddy KN, Pattanaik C, Reddy CS, Raju VS. Traditional knowledge on wild food plants in Andhra Pradesh. Indian J Tradit Knowl. 2007;6(1):223-9.

48. Ngome-Tata P, Shackleton C, Degrande A, Tieguhong JC. Addressing constraints in promoting wild edible plants' utilization in household nutrition: case of the Congo Basin forest area. Agric Food Secur. 2017; https://doi.org/10.1186/s40066-017-0097-5.

49. Chettri N, Sharma E, Lama SD. Non-timber forest produces utilisation, distribution and status in trekking corridor of Sikkim. India J Ecol Appl. 2008;8(1):93-108.

50. Misra S, Maikhuri RK, Kala CP, Rao KS, Saxena KG. Wild leafy vegetables: a study of their subsistence dietic support to the inhabitants of Nanda Devi Biosphere Reserve, India. J Ethnobiol Ethnomed. 2008;4:15.

51. Kaliszewska I, Kolodziejska-Degorska I. The social context of wild leafy vegetables uses in Shiri, Daghestan. J Ethnobiol Ethnomed. 2015;11:63.

52. Kaliszewska D, Degórska K. The social context of wild leafy vegetables uses in Shiri, Daghestan. J Ethnobiol Ethnomed. 2015;11:63.

53. Shrestha PM, Dhillion SS. Diversity and traditional knowledge concerning wild food species in a locally managed forest in Nepal. J Agroforest Syst. 2006;66(1):55-63.

54. Acharya KP, Acharya R. Eating from the wild: indigenous knowledge on wild edible plants in Parroha VDC of Rupandehi district, Central Nepal. Internat J Soc Forest. 2010;3(1):28-48.

55. Gari JA. Agrobiodiversity, food security and HIV/AIDS mitigation in SubSaharan Africa. 2002. http://www.fao.org/fileadmin/templates/esw/esw_ new/documents/Links/publications_other/8_Agrobiodiversity_Gar\%C3\%AD. pdf. Accessed 5 Dec 2016.

56. Harris FMA, Mohammed S. Relying in nature: wild foods in Northern Nigeria Ambio J Human Env. 2003;32(1):24-9.

57. Hunde D, Njoka J, Asfaw Z, Nyangito M. Seasonal availability and consumption of wild edible plants in semiarid Ethiopia: implications to food security and climate change adaptation. J Hortic For. 2011;3:138-49.

58. Zhang L, Chai Z, Zhang Y, Geng Y, Wang Y. Ethnobotanical study of traditional edible plants used by the Naxi people during droughts. J Ethnobiol Ethnomed. 2016:2:39.

59. Panmei R, Gajurel PR, Singh B. Ethnobotany and nutritional values of some selected wild edible plants used by Rongmei tribe of Manipur Northeast India. Internat J Appl Biol Pharmaceut Technol. 2016;7(4):1-9.

60. Konsam S, Thongam B, Handique AK. Assessment of wild leafy vegetables traditionally consumed by the ethnic communities of Manipur, northeast India. J Ethnobiol Ethnomed. 2016;12:9.

61. Grivetti LE, Ogle BM. Value of traditional foods in meeting macro and micronutrient needs: the wild plant connection. Nutr Res Rev. 2000;13(1): $31-46$.

62. Bhandari MR, Kasai T, Kawabata J. Nutritional evaluation of wild yam (Dioscorea spp.) tubers of Nepal. Food Chem. 2003;82(4):619-23.

63. Shrestha N, Prasai D, Shrestha KK, Shrestha S, Zhang XC. Ethnomedicinal practices in the highlands of central Nepal: a case study of Syaphru and Langtang village in Rasuwa district. J Ethnopharmacol. 2014;155(2):1204-13.

64. Shinwari ZK. Medicinal plants research in Pakistan. J Med Plants Res. 2010;4: 161-76.

65. Manandhar NP, Manandhar S. Plants and people of Nepal. Portland: Timber press; 2002

66. Kunwar RM, Bussmann RW. Medicinal plants and quantitative ethnomedicine: a case study from Baitadi and Darchula Districts, Far-West Nepal. J Nat Hist Mus. 2009;24:73-82.

67. O'Neill AR, Badola HK, Dhyani PP, Rana SK. Integrating ethnobiological knowledge into biodiversity conservation in the Eastern Himalayas. J Ethnobiol Ethnomed. 2017;13:21.

68. Kunwar RM, Shrestha KP, Bussman RW. Traditional herbal medicine in Farwest Nepal: a pharmacological appraisal. J Ethnobiol Ethnomed. 2010;6:35 
69. Kunwar RM, Nepal BK, Kshhetri HB, Rai SK, Bussmann RW. Ethnomedicine in Himalaya: a case study from Dolpa, Humla, Jumla and Mustang districts of Nepal. J Ethnobiol Ethnomed. 2006;2:27.

70. Kunwar R, Burlakoti C, Chowdhary CL, Bussman RW. Medicinal plants in farwest Nepal: indigenous uses and pharmacological validity. Med Aromat Plant Sci Biotechnol. 2010;28:42.

71. Negi VS, Pathak R, Chandra Sekar K, Rawal RS, Bhatt ID, Nandi SK, Dhyani PP. Traditional knowledge and biodiversity conservation: a case study from Byans Valley in Kailash Sacred Landscape, India. J Environ Plan Manag. 2017; https://doi.org/10.1080/09640568.2017.1371006.

72. Uddin MZ, Hassan MA. Determination of informant consensus factor of ethnomedicinal plants used in Kalenga Forest, Bangladesh. J Plant Taxon. 2014;21(1):83-91.

73. Pandey NC, Bhatt D, Arya D, Chopra N, Upreti BM, Joshi GC, Tewari LM. Diversity of ethno-medicinal plant: a case study of Bageshwar district Uttarakhand. J Med Plants Stud. 2017;5(2):11-24.

74. Ong HG, Kim YD. The role of wild edible plants in household food security among transitioning hunter-gatherers: evidence from the Philippines. Food Sec. 2017;9(1):11-24.

75. Ashagre M, Asfaw Z, Kelbessa E. Ethnobotanical study of wild edible plants in Burji District, Segan Area Zone of Southern Nations, Nationalities and Peoples Region (SNNPR), Ethiopia. J Ethnobiol Ethnomed. 2016;12(1):32-56.

76. Ahmad K, Pieroni A. Folk knowledge of wild food plants among the tribal communities of Thakht-e-Sulaiman Hills, North-West Pakistan. J Ethnobiol Ethnomed. 2016;12:7.

77. Aryal KP, Kerkhoff EE. The right to practice shifting cultivation as a traditional occupation in Nepal: a case study to apply ILO conventions nos. 111 (employment and occupation) and 169 (indigenous and tribal peoples). Geneva: ILO; 2008

78. Jena MK. Economical and biological potential of Non-Timber Forest Products (NTFP) in the Similipal Hills of Orissa. In: Seeland, Schmithusen, editors. Indigenous knowledge, forest management and forest policy in South Asia. Man and forest series 5; 2003. p. 81-103.

79. Kunwar RM, Bussmann RW. Medicinal, aromatic and dye plants of Baitadi and Darchula districts, Nepal Himalaya: status, uses and management. In: Hartmann M, Weipert J, editors. Biodiversitat und Naturausstattung im Himalaya III. Erfurt: Naturekunde Museum; 2009. p. 43-9.

80. Hickey GM, Pouliot M, Smith-Hall C, Wunder S, Nielsen MR. Quantifying the economic contribution of wild food harvests to rural livelihoods: a globalcomparative analysis. Food Policy. 2016;62:122-32.

81. Kunwar R, Bussman RW. Ethnobotany in the Nepal Himalaya. J Ethnobiol Ethnomed. 2008:4:24.

82. Addis G, Asfaw Z, Woldu Z. The role of wild and semi-wild edible plants in household food sovereignty in Hamer and Konso communities, South Ethiopia. Ethnobot Res Applic. 2013;11:251-71.

83. Smith GC, Clegg MS, Keen CL, Grivett LE. Mineral values of selected plant foods common to Southern Burkina Faso and Niamey, Niger, West Africa. Int J Food Sci Nutr. 1995:47:41-53.

84. Pieroni A, Soukand R. The disappearing wild food and medicinal plant knowledge in a few mountain villages of North-Eastern Albania. J Appl Bot Food Qual. 2017;90:58-67.

85. Bharali P, Sharma M, Sharma CL, Singh B. Ethnobotanical survey of spices and condiments used by some tribes of Arunachal Pradesh. J Med Plants Stud. 2017;5(1):101-9.

\section{Submit your next manuscript to BioMed Central and we will help you at every step:}

- We accept pre-submission inquiries

- Our selector tool helps you to find the most relevant journal

- We provide round the clock customer support

- Convenient online submission

- Thorough peer review

- Inclusion in PubMed and all major indexing services

- Maximum visibility for your research

Submit your manuscript at www.biomedcentral.com/submit
Biomed Central 